\title{
Numerical simulation of droplet impact on wettability-patterned surfaces
}

\author{
Antonio Russo, ${ }^{1,2}$ Matteo Icardi, ${ }^{3}$ Mohamed Elsharkawy, ${ }^{1}$ Diego Ceglia, ${ }^{2}$ Pietro Asinari, ${ }^{2}$ and Constantine M. Megaridis ${ }^{1}$ \\ ${ }^{1}$ Department of Mechanical and Industrial Engineering, \\ University of Illinois at Chicago, Chicago, IL 60607, United States \\ ${ }^{2}$ Energy Department, Politecnico di Torino, Corso Duca degli Abruzzi 24, Torino, 10129, Italy \\ ${ }^{3}$ School of Mathematical Sciences, University of Nottingham, NG7 2RD, United Kingdom
}

(Dated: June 23, 2020)

Numerical simulations have unexplored potential in the study of droplet impact on non-uniform wettability surfaces. In this work, we compare numerical and experimental results to investigate the application potential of a Volume of Fluid method utilized in OpenFOAM®. The approach implements the Kistler model for the dynamic contact angle of impacting droplets. We begin with an investigation on the influence of the most important solver parameters in order to optimize the computational setup and reach the best compromise between computational cost and solution errors, as assessed in comparison to experimental results. Next, we verify the accuracy of the predictions for droplet impact on uniformly hydrophilic or superhydrophobic surfaces. Benchmarking the maximal spreading factor, contact and spreading times, as well as contact-line behavior, we show strong agreement between the present numerical results and the models of Pasandideh-Fard et al. (1996) and Clanét et al. (2004). Lastly, we demonstrate the capability of the model to accurately predict outcome behaviors of droplets striking distributed-wettability surfaces, which introduce 3-D outcome characteristics, even in orthogonal impact. The model successfully predicts droplet splitting and vectoring, as reported in the experiments of Schutzius et al. (2014). Finally, we demonstrate a configuration wherein a spreading droplet becomes arrested within a disc of higher wettability than its surrounding domain. The main contribution of the present work is a numerical model capable of accurately simulating droplet impact on spatially non-uniform wettability patterns of any foreseeable design.

Keywords: droplet impact, wettability-patterned surfaces, dynamic contact angle, Volume of Fluid (VOF), OpenFOAM® 


\section{INTRODUCTION}

Droplet impact on solid surfaces, which is frequently encountered in everyday life, has been an important target of human curiosity and scientific studies dating back hundreds of years. The complexity of the problem lies in the pursuit for deeper understanding of the underlying physical mechanisms (e.g., surface tension, viscous forces, friction and inertia) affecting the dynamics and the manner in which they are interwoven [1-3]. In addition to the impact problem producing fundamental insight into crucial physical phenomena, it is also relevant to a plethora of applications, as for example, ink-jet printing [4, 5], spraying $[6,7]$, anti-icing [8], self-cleaning [9], and anti-corrosion [10].

Over the past several decades, a large number of studies has been conducted on droplets striking solid surfaces. These studies have ranged from experimental to analytical or entirely computational efforts. Several experimental investigations have been performed in order to evaluate the effect of common parameters and discern some empirical relationships [11-13]. These parameters include, but are not limited to: the Weber number, defined as the ratio between inertial and surface tension forces $W e=\frac{\rho U_{\circ}^{2} D_{\circ}}{\sigma}$, where $\rho, U_{\circ}, D_{\circ}$ are the density, impact velocity, and droplet diameter, respectively, and $\sigma$ is the liquid surface tension; the Reynolds number, which is the ratio of inertial and viscous forces $R e=\frac{\rho U_{\circ} D_{\circ}}{\mu}$, where $\mu$ is the dynamic viscosity; the Ohnesorge number, which relates viscous, inertial and surface tension forces $O h=\frac{\mu}{\sqrt{\rho \sigma D_{\circ}}}=\frac{W e}{\sqrt{R e}}$; the Eotvos Number, $E o=\frac{\Delta \rho g D_{\circ}^{2}}{\sigma}$, which compares gravitational and surface-tension forces, $\Delta \rho$ is the difference in density between the two phases, and $g$ the gravitational constant; the Capillary number, $C a=\frac{\mu U_{\circ}}{\sigma}$, or ratio of viscous and surface tension forces. The interaction between the solid surface and the two fluids (liquid, gas) is commonly described by the so-called "contact angle" $\theta_{\circ}$, a parameter derived from the equilibrium among the liquid, solid and vapor interfaces.

Several analytical models have been put forth in attempts to predict droplet-impact outcome. These models generally attempted to describe the spreading and receding behavior of impacting droplets depending on the aforementioned dimensionless parameters. The majority of these models were derived by considering the energy changes of the system, with several geometric assumptions made for the shape of the spreading droplets. Some of the most successful and well-known models are those of Pasandideh-Fard et al. [14], Scheller and Bousfield [15], Roisman [16] and Clanét et al. [17].

As insightful as prior reports have been, the complexity of droplet impact cannot be fully comprehended only by experimental and analytical techniques. A need still exists for the use of high-performance computational tools in unraveling the physics of droplet impact. In fact, a large body of work has reported computational and numerical approaches of droplet impact on spatially-uniform solid surfaces. For comprehensive reviews of experimental, analytical, and computational studies in this area, the reader is referred to Yarin [18], and Josserand and Thoroddsen [3].

The vast majority of prior studies reported in the literature primarily concerned droplet impact on surfaces with spatiallyuniform wettability. Little experimental or analytical work concerned droplet impact on non-uniform wettability surfaces (also known as wettability-patterned surfaces) and even less so on computational/numerical approaches addressing the matter. Nonuniform wettability surfaces are primarily sorted in two categories: multi-region wettability surfaces (MRS), and gradientwettability surfaces (GWS). MRS [19-27] comprise solid substrates with spatial domains of distinct wettabilities placed adjacent to one another with clear boundaries. On the other hand, GWS [28-30] are surfaces with a gradient in wettability properties due, for instance, to a continuous variation in roughness. These two kinds of non-homogeneous surfaces allow for passive control of the droplet impact dynamics and can be designed to cause specific outcomes. As important -and relevant- as these surfaces are, studies of droplet impact on such surfaces in the computational realm [31] are rare, with very few numerical reports [3234] addressing such non-uniform surfaces. For instance, in a the recent work Xu [35] et al. studied numerically the droplet impact on an hydrophobic surface patterned with hydrophilic dots. A Volume of Fluid method has also been adopted to study hydrophilic surfaces decorated with a hydrophobic strip in Ref. [36]. In addition, recent reports in the area of impact on nonuniform surfaces have demonstrated the limitless opportunity of complex physics that can extracted from these type of surfaces. Li et al.[37] showed that the translational motion of an impacting droplet can be converted to gyration with a proper wettabilitypatterned surface. Such complex physics cannot intuitively be derived. The physical opportunities that these surface provides are limitless and require proper investigation. Thus there is a need to develop a comprehensive, holistic method capable of studying these surfaces.

To fill this gap, the main objective of the present work is to develop a numerical capability to analyse droplet impact on solid surfaces, with special attention to wettability-patterned domains. This capability is developed using OpenFOAM®, an opensource computational fluid dynamics code [38-40]. In particular, the present work comprises an investigation on the influence of the most important OpenFOAM® solver parameters towards achieving the best compromise between computational power and solution errors, as assessed in comparison to experimental results. In addition, a study of the so-called "spurious currents," i.e. non-physical phenomena often plaguing numerical simulations, is performed in order to limit their adverse effects on the model predictions. First, we use previously-reported models to benchmark our numerical predictions for impact on uniform surfaces. Upon demonstrating proper agreement, we expand the method to accommodate a multitude of wettability-patterned surfaces. Specifically, the model is shown to accurately reproduce two important physical phenomena: 1) Droplet Splitting [31, 41], and 2) Droplet vectoring [42, 43]. Splitting consists of dividing an impacting liquid droplet into multiple volumes through 
a combination of hydrophilic and superhydrophobic target regions [25]. Vectoring is a directed rebound of the droplet after orthogonal impact, passively guided by the non-uniform wettability of the patterned surface [25, 28]. Using available results, we show good agreement between experimental droplet impact on non-uniform wettability surfaces and the present numerical simulations. We conclude that even though the present study is confined to a limited number of wettability designs, the present techniques are amenable to any conceivable wettability-patterned surface.

\section{NUMERICAL MODEL}

\section{A. Algebraic Volume of Fluid method}

Due to the multiphase nature of droplet impact, numerical simulations require an interface tracking method, such as Marker and Cell (MAC) [44, 45], Volume of Fluid (VOF) [46-50] or Level Set (LSM) [51, 52], with some studies making use of Finite Elements applied to moving grids [53] and Lattice Boltzmann methods [54, 55]. In the present work, a modified version of the VOF method, initially developed by Hirt and Nicholson [56], is employed considering its ability to handle severe interface deformations. The method, based on a fully-Eulerian formulation, features the transport equation of an indicator function called "phase fraction", $\alpha$, together with the continuity and momentum equations. The transport equations for incompressible fluids are considered in the following forms

$$
\begin{gathered}
\frac{\partial \alpha}{\partial t}+\nabla \cdot(\mathbf{U} \alpha)=0 \\
\nabla \cdot \mathbf{U}=0 \\
\frac{\partial \rho \mathbf{U}}{\partial t}+\nabla(\rho \mathbf{U} \otimes \mathbf{U})=-\nabla p++\nabla \cdot\left[\mu\left(\nabla \mathbf{U}+(\nabla \mathbf{U})^{T}\right)-\frac{2}{3} \mu(\nabla \cdot \mathbf{U}) \mathbf{I}\right]+\rho \mathbf{a}
\end{gathered}
$$

where $\mathbf{U}$ is the velocity vector shared by the fluids (different fluids occupying the same volume element are assumed to have the same velocity), $p$ is the pressure, I the identity matrix, a the external force per unit mass, such as gravitational acceleration (source term), and $\alpha$ within each cell of the mesh designates how much of the volume in this cell is occupied by each fluid. Consequently, $\alpha$ is a scalar function defined inside the simulation domain and varying between zero (i.e., all vapor) and one (i.e., all liquid). With this quantity and the assumption of immiscible fluids, the numerical model considers the two fluids in each cell as a single medium with physical properties computed by an $\alpha$-weighted average of the constituent properties. Thus, density and viscosity of the equivalent fluid are computed by $\rho=\rho_{l} \alpha+\rho_{v}(1-\alpha)$ and $\mu=\mu_{l} \alpha+\mu_{v}(1-\alpha)$, where the subscript $l$ refers to the liquid, and $v$ to the vapor.

The OpenFOAM@-based solver, interFoam, implements a modified two-phase formulation of the VOF method. This new formulation gives rise to an additional convective term, which is due to the representation of the velocity terms as weighted averages. The full derivation of this convective term begins by solving the phase fraction equations separately,

$$
\begin{gathered}
\frac{\partial \alpha}{\partial t}+\nabla \cdot\left(\mathbf{U}_{l} \alpha\right)=0 \\
\frac{\partial(1-\alpha)}{\partial t}+\nabla \cdot\left(\mathbf{U}_{v}(1-\alpha)\right)=0
\end{gathered}
$$

where $l, v$ signify the liquid and vapor phases, respectively. The velocity of the equivalent fluid is considered to be a weighted average of the two velocities, $\mathbf{U}=\mathbf{U}_{l} \alpha+\mathbf{U}_{v}(1-\alpha)$. Combining these terms together yields,

$$
\frac{\partial \alpha}{\partial t}+\nabla \cdot(\mathbf{U} \alpha)+\nabla \cdot\left(\mathbf{U}_{r} \alpha(1-\alpha)\right)=0
$$

where $\mathbf{U}_{r}=\mathbf{U}_{l}-\mathbf{U}_{v}$, is the relative velocity between the two fluids.

In regards to the surface tension, its effect is considered inside the momentum equation by adding the source term $\mathbf{f}_{\sigma}$ evaluated according to the Continuum Surface Model introduced by Brackbill et al. [57]. Thus $\mathbf{f}_{\sigma}=\sigma \kappa \nabla \alpha$, where $\kappa$ is the mean curvature, evaluated as $\kappa=-\nabla \cdot \mathbf{n}$. Far from the wall, the unit vector normal to the free interface is computed by $\mathbf{n}=\nabla \alpha /|\nabla \alpha|$. 
Furthermore, for convenience in the setup of the boundary conditions, the hydrostatic component of the pressure is subtracted so that a modified quantity, $p_{d}=p-\rho \mathbf{g} \cdot \mathbf{h}$, where $p$ is the pressure of the droplet and $\mathbf{h}$ the depth, is adopted. Consequently, the momentum equation becomes

$$
\frac{\partial \rho \mathbf{U}}{\partial t}+\nabla(\rho \mathbf{U} \otimes \mathbf{U})=-\nabla p_{d}-\mathbf{g} \cdot \mathbf{h} \nabla \rho+\nabla \cdot\left[\mu\left(\nabla \mathbf{U}+(\nabla \mathbf{U})^{T}\right)\right]+\mathbf{f}_{\sigma}
$$

The last relationship completes the system and allows to solve for $\mathbf{U}_{\mathrm{r}, \mathrm{f}}$, i.e. $\mathbf{U}_{\mathrm{r}}$ evaluated at each face from the following relation [58]

$$
\mathbf{U}_{\mathrm{r}, \mathrm{f}}=\min \left[C_{\alpha}\left|\frac{\phi}{\left|\mathbf{S}_{f}\right|}\right|,\left|\frac{\phi}{\left|\mathbf{S}_{f}\right|}\right|_{\text {max }}\right]
$$

where $\phi$ is the face flux, $\mathbf{S}_{f}$ is the face area vector, $C_{\alpha}$ a constant $\left(C_{\alpha}=1\right.$ in this case $)$ and $\left|\frac{\phi}{\left|\mathbf{S}_{f}\right|}\right|_{\text {max }}$ is the maximum phase flux velocity in the flow field. It is worth noticing that the compression term contributes only for $0<\alpha<1$, i.e. only at the interface, and limits the error due to interfacial smearing, enhancing the convergence of the scheme for fine meshes [58]. Moreover, in line with previous works, a value of the parameter $C_{\alpha}$ equal to unity is adopted, since increasing or decreasing it was found to exacerbate errors in interfacial curvature and interfacial smearing (the reader is referred to Refs $[39,58]$ and references therein for a comprehensive discussion on this term).

\section{B. Discretization and MULES/PISO parameters}

The discretization approach is the well-established finite volume method [59-61], which subdivides the flow domain into a finite number of smaller nonoverlapping control volumes that completely fill the domain. In particular, a co-located arrangement of the variables, stabilized by the Rhie and Chow velocity interpolation method [62], is implemented in OpenF0AM ®[63]. The discretization of Equations 6 and 7 requires specific schemes for the transient and spatial terms. All spatial derivative terms are converted to integrals over the cell surface, bounding the volume using Gauss's theorem [39], while an Euler implicit scheme is used for the transient terms. Following this, interpolation between neighboring cell-center quantities is required to find values at the face centers.

The advection terms in Equation 6 require special treatment in order to ensure that $\alpha$ stays between 0 and 1 . This task is made by the interFoam solver MULES, a Flux Corrected Transport technique that maintains boundedness of $\alpha$ regardless of underlying numerical scheme and mesh structure [39, 40]. A prudential approach was adopted, choosing a bounded Van Leer interpolation scheme [64, 65] for the linear term in Equation 6, and a linear scheme for the quadratic term in the same equation.

The Pressure Implicit with Splitting of Operators (PISO) algorithm [66] was adopted to handle the pressure-velocity coupling. In this approach, the equations of the system are solved one-by-one in a loop, seeking convergence of the solution. The PISO algorithm is widely used and detailed descriptions of its implementation can be found in Versteeg and Malalasekera [60], and Ferziger and Peric [67].

\section{Boundary conditions}

The most commonly used parameter to characterize the wettability of a surface by a specific liquid is the equilibrium contact angle, $\theta_{0}$, which is defined as the angle between the liquid/solid and liquid/gas interfaces at the three-phase sessile contact line. For ideal surfaces under equilibrium conditions, the value of the contact angle $\theta_{0}$ can be found using Young's equation [68]. However, for real (non-ideal) surfaces, it has been verified that the contact angle varies over a certain range. The upper and lower limits of this range are known as the advancing contact angle $\left(\theta_{a}\right)$ and receding contact angle $\left(\theta_{r}\right)[69,70]$, respectively. In addition, it has been observed experimentally that $\theta_{a}$ and $\theta_{r}$ are functions of the Capillary number at the contact line [69]. For this reason, the so-called dynamic contact angle, $\theta_{d}$, has been introduced. In order to impose a boundary condition that yields an accurate approximation of the dynamic behavior of the contact angle, we have tested several dynamic contact angle models and adopted the one by Kistler [71]. In this model, the dependence of the generic contact angle upon the contact-line speed $U_{c l}$, can be evaluated through the following expressions

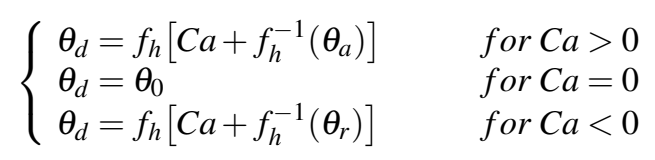


TABLE I. FLUID PHYSICAL PROPERTIES

\begin{tabular}{ll} 
Property & \multicolumn{1}{l}{ Value } \\
\hline Water density & $1000 \mathrm{~kg} \mathrm{~m}^{-3}$ \\
Air density & $1 \mathrm{~kg} \mathrm{~m}^{-3}$ \\
Surface tension & $0.07 \mathrm{~N} \mathrm{~m}^{-1}$ \\
Water kinematic viscosity & $10^{-6} \mathrm{~m}^{2} \mathrm{~s}^{-1}$ \\
& \\
Air kinematic viscosity & $1.48 \times 10^{-5} \mathrm{~m}^{2} \mathrm{~s}^{-1}$
\end{tabular}

where $C a$ is evaluated at the contact line, and $f_{h}$ is Hoffman's function [72] defined by

$$
f_{h}(x)=\cos ^{-1}\left\{1-2 \tanh \left[5.16\left(\frac{x}{1+1.31 x^{0.99}}\right)^{0.706}\right]\right\}
$$

While the above choice of the functional form of the dynamic contact angle may not be universally accommodating, it is made here as one that produced reliable results.

The implementation of the contact angle as boundary consition follows the approach in Brackbill et al. [57]. The unit vector normal to the interface $\boldsymbol{n}$ is evaluated as:

$$
\boldsymbol{n}=\boldsymbol{n}_{w} \cos \theta_{d}+\boldsymbol{n}_{t} \sin \theta_{d}
$$

with $\boldsymbol{n}_{w}$ and $\boldsymbol{n}_{t}$ being the unit vectors respectively normal and tangential to the wall. The unit vector $\boldsymbol{n}$ is then used to compute the curvature and the surface tension force $\mathbf{f}_{\sigma}$ in the cells at the boundaries, as discussed in the previous sections.

\section{COMPUTATIONAL AND P HYSICAL PARAMETERS}

The physical properties for water and air (room temperature and $1 \mathrm{~atm}$ ) used in the simulations are listed in Table I. The release height necessary to reach a drop impact velocity of $1.18 \mathrm{~ms}^{-1}$ is $\sqrt{\frac{v^{2}}{2 g}} \approx 71 \mathrm{~mm}$, implying the need for a large computational domain and a high computational cost. Thus, we explored alternative avenues to simulate an impact event. We compared the results of various techniques in order to select the most physically-realistic method for modeling the impact.

\section{A. Computational Domain}

The computational domain is simplified by taking advantage of the symmetries (when present) of the event; such simplification is necessary due to limits in computational power. Axial-symmetric impact cases are modelled with a wedge-like shape domain with a single cell along one direction. For more complex cases that lack axisymmetry, other symmetry planes are exploited to reduce the computational grids. Preliminary tests showed that when the expanding liquid rim got close to the border of the computational domain, assuming that symmetric boundary conditions were met, the interactions with the boundary affected the results. For this reason, the domain size was chosen to be, depending on the simulated configuration, large enough to prevent any influences from the outer boundaries.

\section{B. Initial conditions}

The main parameter that affects the impact most drastically is the impact velocity, defined as the velocity of the center of mass of the droplet upon first contact with the stationary solid. However, the local state of the fluid and the interface could be 
significantly different even with the same impact velocity. In a real-world scenario, this state would be dependent on the motion within the droplet before impact [73]. Most of the computational studies on droplet impact have assumed an initial condition (extremely close to the surface) with a predetermined impact velocity imposed uniformly on the liquid phase. Here, instead, an attempt was made to include the droplet internal motion before impact by releasing the droplet from different heights and always with zero velocity.

To investigate the height-release effect, we first considered droplet impact with $U_{\circ}=1.18 \mathrm{~ms}^{-1}$, as in Rioboo et al. [74]. Under earth's gravity, the droplet has to be released from a height $h \sim 71 \mathrm{~mm}$, which translates into a prohibitively large simulation domain. To avoid this issue and analyze pre-impact effects, we split the simulation in two stages: a first stage with increased gravitational acceleration during the falling phase and a second stage with real gravity acting during the impact phase. In order to preserve the physics of the phenomenon in the modified configuration, we maintain the non-dimensional numbers involving gravity, constant. Under these circumstances, we explored impact events in two alternative configurations: a) with a constant Eotvos number, and b) with constant Weber and Capillary numbers. In the first case, both $g$ and $\sigma$ were multiplied by a factor $K$ ( $K=17$ in this case), while in the second case $g, \sigma, \rho$ and $\mu$ were multiplied by the same factor $K$. The comparison between the two cases in Figure 1a,b shows the presence of non-physical deformation at the end of the falling stage in the case of constant Eo. This is due to the appearance of the so-called "spurious currents," numerical artifacts that appear at the fluid interface when the discrete balance is not verified and/or the interface curvature is not computed accurately [75-77]. These currents are continuously supplied by the numerical imbalance and are bounded only by viscous dissipation. If viscous dissipation is low and surface tension is high, as in the case of constant $E o$, the currents can reach severely high values, affecting the predicted shape of the droplet (see Supplemental Material [78] and Harvie et al. [79] for more details). In relation to the two configurations tested, constant $\mathrm{We}$ and $\mathrm{Ca}$ yield more physically-sound results.

Subsequently, a simpler approach was tested, with the impacting droplet positioned a few millimetres above the solid with the specified impact velocity. The results were compared against the previous two-stage approach with constant $W e$ and $\mathrm{Ca}$. It is a foreseeable consequence that the shear (from the surrounding air) during the fall could produce internal water circulation $[73,80]$ that may affect the shape evolution of the droplet at impact. Comparing these two circumstances, however, as Figure 1c shows, no relevant effects on the shape, impact, or spreading behavior of the impacting droplets were observed. Thus, in order to reduce the computational intensity, for all simulations reported below, all impact events were simulated with droplets released from a few millimetres above the solid floor with a specified and uniform impact velocity.

\section{Solution convergence}

In order to reach a compromise between solution accuracy and execution time, a preliminary study on the main solver parameter was performed and the convergence of the solution was verified by refining the adopted computational meshes. Specifically, we focused on the parameters of the MULES and PISO algorithms introduced earlier and the convergence of the pressurevelocity coupling algorithm PIMPLE, which iterates over the MULES and the PISO solvers (more details on the meaning of the parameters and the setup are provided in the Supplementary Material [81] and Ubbink [82]).

In addition, convergence of the numerical results with respect to global mesh refinement was studied. The simulation requires the transient analysis to be performed at every time step, each of the order of $10^{-6}$ seconds, while the entire event lasts for about $10^{-2}$ seconds. Due to the extensive resources needed to model these phenomena, a compromise had to be reached between accuracy and computational time. Figure 2 plots the total error for each mesh with a number of cells 'i' as $\varepsilon_{t o t}=\sum_{t}\left|r_{t}{ }^{i}-r_{t}{ }^{i_{\max }}\right|$, where $i_{\max }$ corresponds to the most refined mesh, as a function of the number of mesh cells. The orders of convergence $n$ observed for the wedge-shape and the full-3D domain are about 1.9 and 1.4, respectively. In the following, we adopted meshes with $\approx 10^{5}$ cells for the wedge-shaped domains, and between $\approx 10^{6}-10^{7}$ for full-3D domains, depending on the impact surface.

\section{Computational resources}

Because of the large number of cells required, the simulation was carried out using the openMPI implementation of MPI (Message Passing Interface). The mesh and fields are decomposed using the decomposePar utility, employing the Scotch decomposition, which requires no geometric input from the user and attempts to minimize the number of processor boundaries [39]. Specifically, the numerical computations have been performed on a supercomputer with 17 nodes, each with 16 cores characterized by 2x AMD Opteron 6276/6376 (Bulldozer) $2.3 \mathrm{GHz}$ (turbo $3.0 \mathrm{GHz}$ ). The jobs have been parallelized among 16 and 128 cores with processing time ranging between 10 to 300 processor hours. 


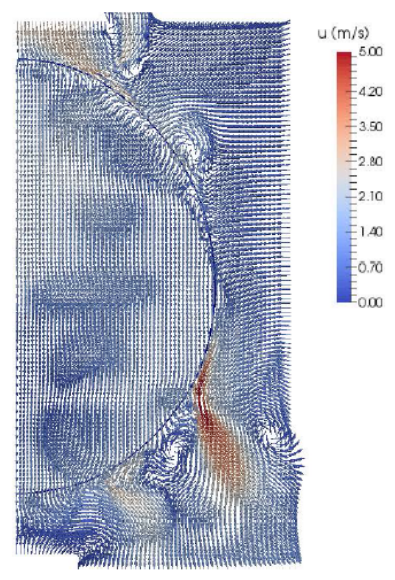

Time: $1 \mathrm{~ms}$

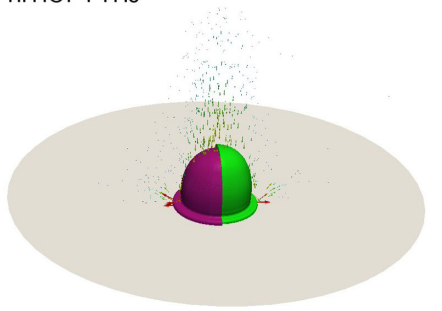

Time: $9 \mathrm{~ms}$

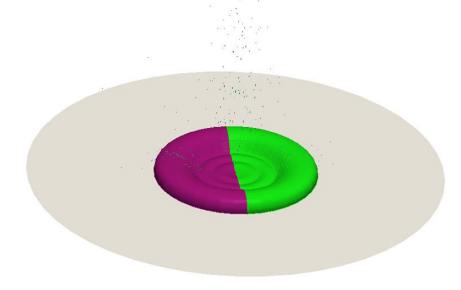

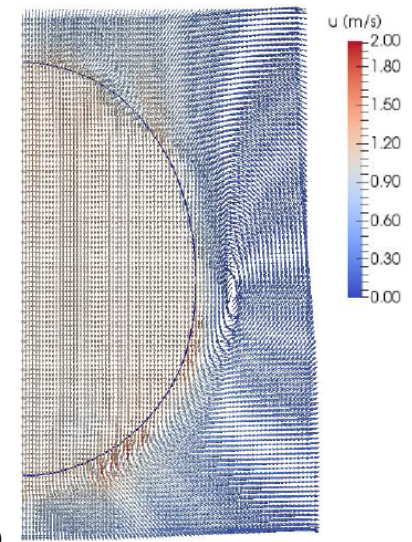

(b)

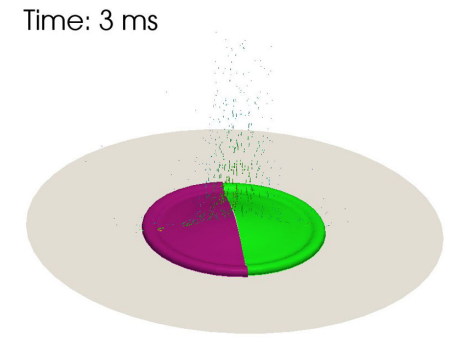

Time: $13 \mathrm{~ms}$

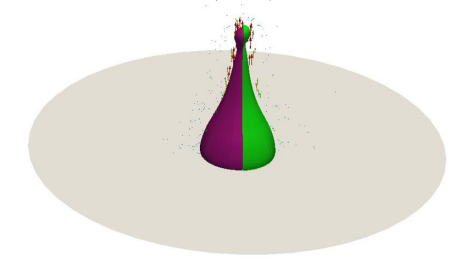

(c)

FIG. 1. Effects of the "spurious currents" at the end of the falling stage for droplet impact with $D_{\circ}=2.75 \mathrm{~mm}$ and $U_{\circ}=1.18 \mathrm{~ms}^{-1}$ in two cases: (a) When the Eotvos number is kept constant, and (b) when the Weber and Capillary numbers are constant. In (c), we compare the shapes of a droplet falling at constant Weber and Capillary numbers (purple) and a droplet released from a few millimeters above the solid, i.e. without falling stage (green). There is little to no difference between the two shapes.

\section{RESULTS AND DISCUSSION}

\section{A. Validation: Impact on uniform-wettability surfaces}

In order to establish the validity of the numerical approach, simple homogeneous flat surfaces were used first to test the accuracy of the simulation results with respect to established relations found in the literature. We focused first on two types of surfaces, namely all-hydrophilic and all-superhydrophobic. Due to the axially-symmetric nature of this problem, simulations were carried only along the $\mathrm{x}-\mathrm{y}$ plane of the wedge domain configuration ( $\mathrm{x}$ is the radial distance from the axis, $\mathrm{y}$ is along the symmetry axis).

Equilibrium, advancing and receding contact angles of the surfaces are input parameters to the code, and designate the wettability of each surface. In addition, droplets with different sizes but equivalent impact velocities are simulated. We maintain We between 20 and 60 in order to adhere to conditions found in the literature. The main parameters reported in the literature and used to gauge our simulations are spreading contact line characteristics, maximum spreading diameter, contact time, and average receding velocity. 


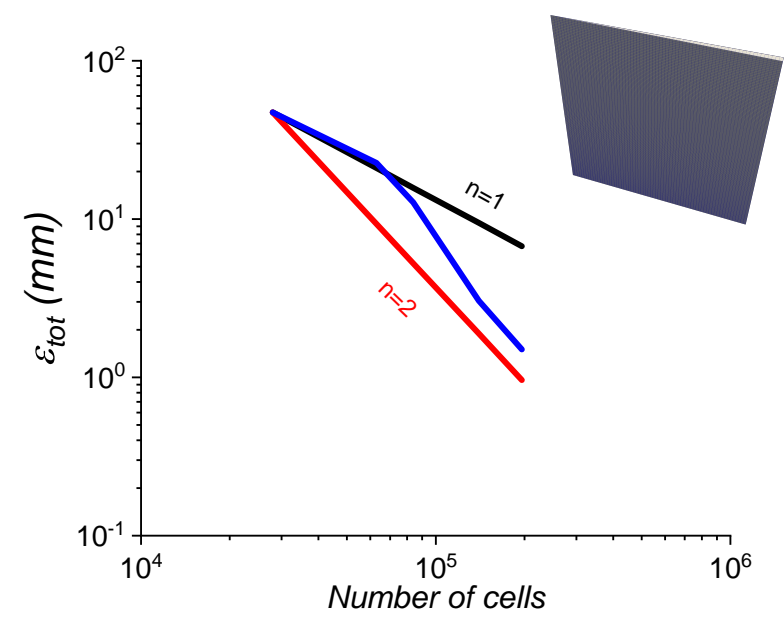

(a)

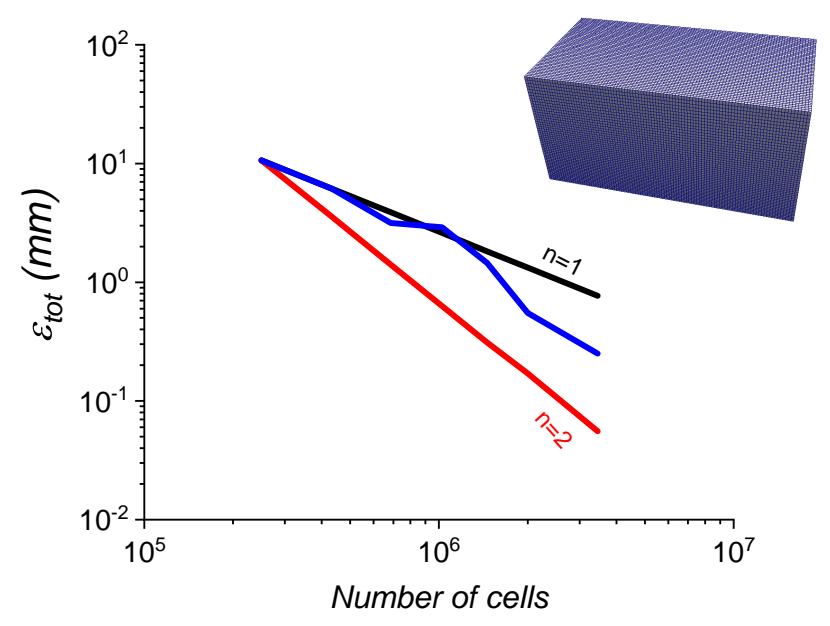

(b)

FIG. 2. Total error $\varepsilon_{t o t}$ (blue line), computed as the sum in time of the absolute differences between the spreading radii obtained for each mesh and the most refined one, vs. total number of mesh cells for: (a) wedge-shaped meshed domain (inset), and (b) full-3D meshed domain (inset). $\varepsilon_{t o t}$ is larger for the wedge-shaped domain, but its order of convergence $(n)$ is higher compared to the full-3D meshed domain. The black and red lines in each graph correspond to the first $(n=1)$ and second $(n=2)$ orders of convergence, respectively.

\section{Impact on hydrophilic surfaces}

A simple homogeneous surface was used first to test the agreement of the predicted results with relations found in the literature. Here, we focus on a uniformly-hydrophilic surface that, due to the axially-symmetric nature of the problem, has been simulated by equations cast only along the $\mathrm{x}-\mathrm{y}$ plane in the wedge configuration.

First, we perform a simulation of a droplet with $D_{\circ}=2.0 \mathrm{~mm}$, impacting with $U_{\circ}=0.2 \mathrm{~ms}^{-1}$ on a surface with equilibrium, advancing and receding contact angles equal to $60^{\circ}$. As shown in Figure 3a, the simulation demonstrates the classical sequential features of impact, spreading and receding. The predicted contact-line position as a function of time (Figure 3c) was compared with both experimental and simulation data of Malgarinos et al. [46] for a similar impact scenario. Our numerical results are in agreement with the data in the literature. Only for the final portion of the receding phase, the simulation demonstrated a slight delay in the receding.

Simulations with different impact droplet sizes and same initial velocities were also conducted. The corresponding Weber numbers ranged between 20 and 51 . We begin by comparing the numerically-derived values of the spreading factor $\left(\xi_{\max }=\frac{D_{\max }}{D_{\circ}}\right)$ against those obtained by using the relations derived in Pasandideh-Fard et al. [14], Scheller and Bousfield [15], Roisman [16], Ukiwe and Kwok [83], and Wildeman et al. [84]. As seen in Figure 4, the predictions are noticeably different from model to model. Our numerical results match quite well with the predicted $\xi_{\max }$ of Pasandideh-Fard et al. [14], expressed as $\xi_{\max }=\sqrt{\frac{W e+12}{3\left(1-\cos \theta_{\mathrm{a}}\right)+4 W e R e^{-1 / 2}}}$. This model not only takes into consideration We and $R e$, but also makes use of the advancing contact angle, $\theta_{\mathrm{a}}$ in predicting $\xi_{\max }$. We also compared the maximum spreading time determined from the present numerical simulation to that predicted by Pasandideh-Fard et al. [14], namely $t_{\mathrm{sp}}=\frac{8 D_{\circ}}{3 U_{0}}$. As seen in Figure $4 \mathrm{~b}$, there is strong agreement between the two models. The present results not only confirm the accuracy and reliability of our technique, but also help reinforce the legitimacy of Pasandideh-Fard et al.'s model in its predictive capability of droplet impact on uniformly-hydrophilic surfaces.

\section{Droplet impact on superhydrophobic substrates}

Next, we perform a similar set of runs to validate the numerical predictions of droplet impact on uniformly-superhydrophobic surfaces. We first considered a droplet with $D_{\circ}=2.1 \mathrm{~mm}, U_{\circ}=0.5 \mathrm{~ms}^{-1}$ striking a surface with equilibrium, advancing and receding contact angles equal to $150^{\circ}$. As shown in Figure 5a, the simulation demonstrates the classical sequential behavior of impact, spreading, receding, and rebound. The predicted contact-line position as a function of time (Figure 5c) was compared with the experimental data of Schutzius et al. [25] for a similar impact scenario. Besides a small asymmetry of the experimental droplet impact, not evident in the simulation, numerical results are in accordance with the experiment. Only for the final portion of the receding phase, the simulation demonstrated a slight delay in the rebound. 


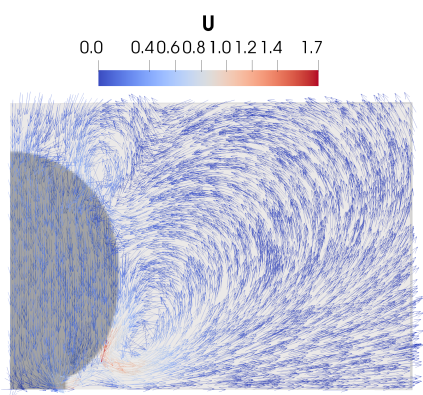

$0.5 \mathrm{~ms}$

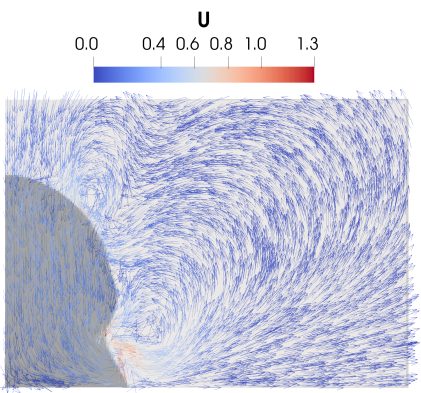

$1.5 \mathrm{~ms}$

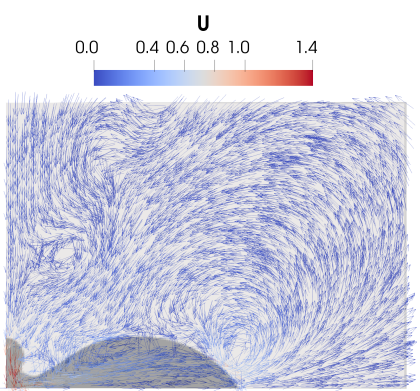

$6.0 \mathrm{~ms}$

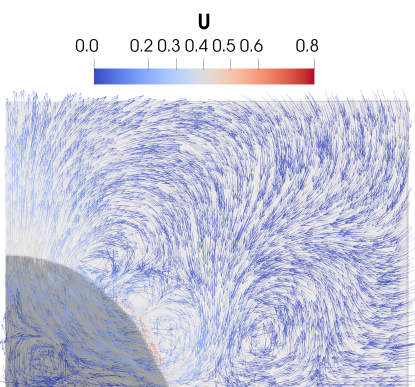

$12.0 \mathrm{~ms}$

(a)

U

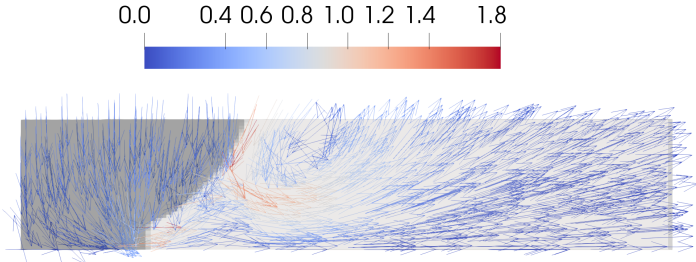

$0.5 \mathrm{~ms}$

U

$\begin{array}{lllllllll}0.0 & 0.4 & 0.6 & 0.8 & 1.0 & 1.2 & 1.4 & 1.8\end{array}$

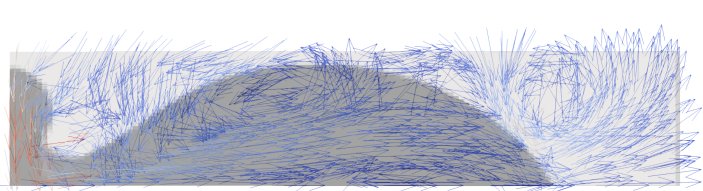

$6.0 \mathrm{~ms}$
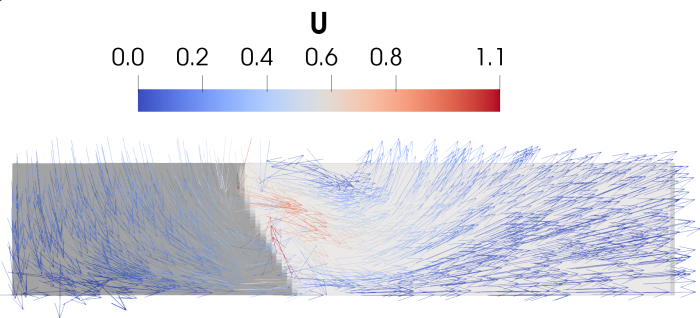

$1.5 \mathrm{~ms}$

$$
\text { U }
$$
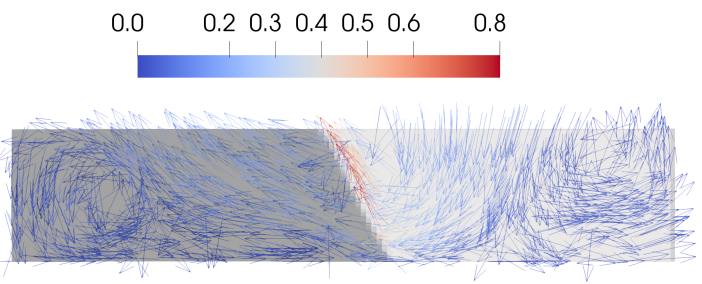

$12.0 \mathrm{~ms}$

(b)

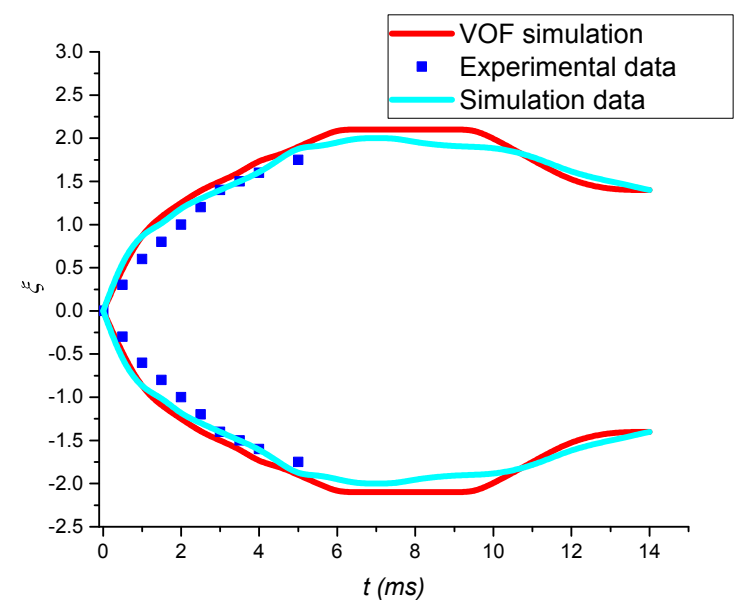

(c)

FIG. 3. (a) Simulated dynamic droplet shapes with $D_{\circ}=2.0 \mathrm{~mm}, U_{\circ}=0.2 \mathrm{~ms}^{-1}$, and $\theta_{0}=\theta_{a}=\theta_{r}=60^{\circ}(W e=1.1$ and $R e=400)$. The droplet shapes demonstrate the expected features of impact, namely, spreading and receding. (b) Velocity field near the contact line. (c) The simulation contact-line position (Red line) vs. time is compared to independently-reported experimental and simulation results under similar impact conditions. The graph shows good agreement of the data sets; the experimental and simulation data were obtained from Malgarinos et al. [46]. 


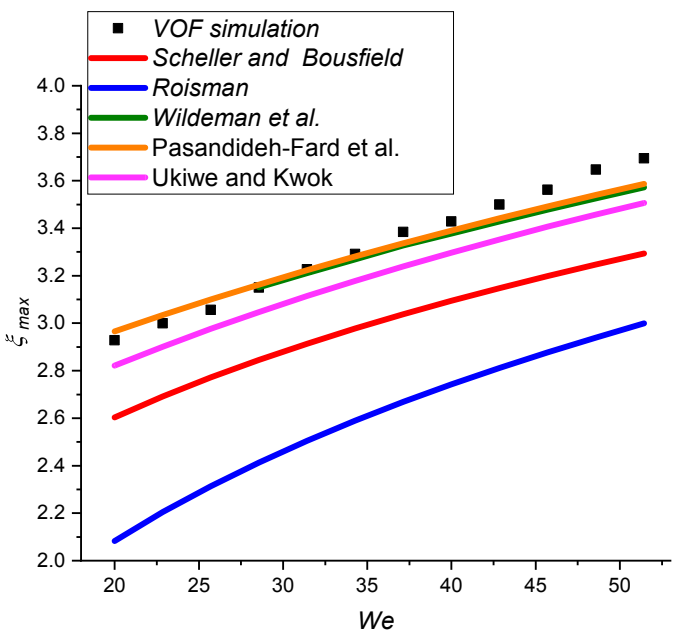

(a)

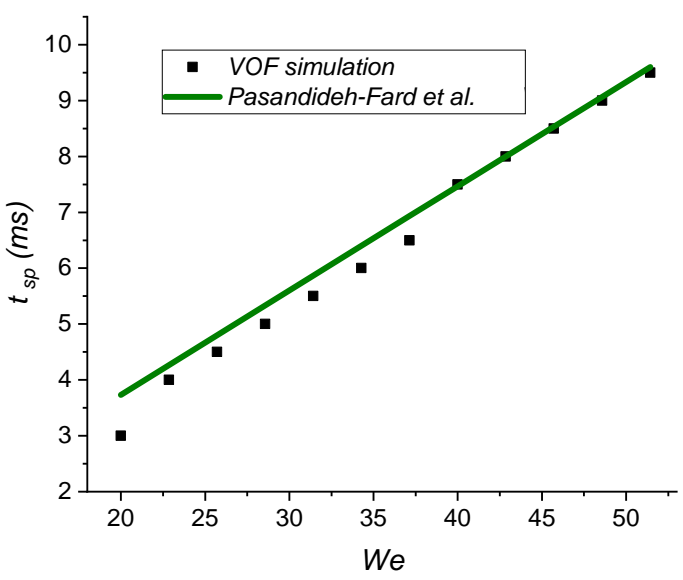

(b)

FIG. 4. Droplets impacting on a hydrophilic surface with contact angles $\theta_{0}=\theta_{a}=\theta_{r}=60^{\circ}$. (a) Comparison of the numerically-predicted maximum spreading factor $\left(\xi_{\max }\right)$ at an impact velocity $U_{\circ}=1.18 \mathrm{~ms}^{-1}$ for droplets of varying initial diameter. The present simulation results (black squares) are compared to the models of Scheller and Bousfield [15] (red line), Roisman [16] (blue line), Wildeman et al. [84] (green line), and Pasandideh-Fard et al. [14] (orange line), and Ukiwe and Kwok [83] (pink line). This graph shows strong agreement between the present simulation results and the model of Pasandideh-Fard et al. [14] and Wildeman et al. [84]. (b) Numerically-predicted spreading times $\left(t_{\mathrm{sp}}\right)$ compared to those of Pasandideh-Fard et al. [14].

Figure 5d plots the average droplet velocity $U_{\text {avr }}=\frac{1}{V} \int_{V} \alpha \mathbf{U} d V$, where $V$ is the droplet volume and $\alpha$ the liquid phase fraction. After the early spreading phase where a linear trend is observed $U_{\text {avr }} \propto t$, the droplet velocity remains null (metastable equilibrium at maximum spreading) for about $1 \mathrm{~ms}$. After this, the velocity starts increasing again in the rebound stage, exhibiting once again a linear trend $U_{a v r} \propto t$ but with a lower slope, ending with a plateau. After complete detachment from the solid substrate -where the maximum value of $U_{a v r}$ is attained- the droplet velocity starts decreasing again under the combined detracting effects of drag and gravity.

To provide further validation, we also analyzed the maximum drop spreading factor. We compared the results from the simulation (Figure 6a) of varying-diameter droplets impacting with the same velocity, to the models of Pasandideh-Fard et al. [14] and Clanét et al. [17]. The predictive equation of Pasandideh-Fard et al. is the same as previously stated for hydrophilic surfaces, while Clanét et al. derived an equation of the form of $\xi_{\max } \sim W e^{\frac{1}{4}}$. As seen from Figure 6a, the present simulation results bare a stronger agreement with the Clanét et al. model than the Pasandideh-Fard et al. prediction. On average, there is a 13\% difference between Pasandideh-Fard et al. and the present simulation, while there is only an average 6\% difference from Clanét et al. This is in agreement with Figure 2 of Clanét et al. [17], where a similar behavior was observed for the experimental data. The model of Clanét et al. was specifically derived for impact on superhydrophobic surfaces and low-viscosity fluids (such as water). Thus, it is reasonable to expect that the present simulation is in better agreement with this model.

We also compared the predicted contact time $t_{c}$, i.e. the time during which droplet and surface remained in contact, with those predicted by Richard $e t$ al. [85]. The contact time, $t_{c}$, is a finite number only for droplet impacts having a rebound and, for this reason, it can be evaluated only in the analysis of droplet impact on superhydrophobic surfaces. According to Richard et al. [85], contact time of a bouncing drop can be approximately computed as $t_{c}=k_{c} \sqrt{\frac{\rho D_{0}^{3}}{8 \sigma}}$, where $k_{c}$ is a constant. Based on this equation, $t_{c}$ is independent of the impact velocity, and dependent on the impact diameter. Thus, for constant-diameter droplets impacting at varying $W e, t_{c}$ is expected to stay constant. In contrast, for constant impact velocity but increasing diameter droplets, $t_{c}$ is expected to increase. Both sets of circumstances have been studied. Figure $6 \mathrm{~b}$ plots cases where the impact velocity was held constant but the diameter was varied. We observe the expected trend, with $k_{c}=2.7 \pm 0.2$ [86].

Another comparison was conducted on the droplet retraction velocity $U_{r e c}$ during the receding phase. According to Bartolo et al. [87], a proportionality exists between the average receding velocity and the impact velocity. Figure $6 \mathrm{~b}$ also presents $U_{\text {rec }}$ for simulations at constant $U_{0}$ and varying initial radius $R_{0}$; as expected, $U_{r e c}$ showed an almost flat trend with We, attaining values of $20-30 \%$ of the impact velocity $U_{0}$.

Based on the above agreement between the theoretical predictions of others and the present numerically-obtained trends, it is within reason to assume validity of our numerical simulation for droplet impact on superhydrophobic surfaces. 


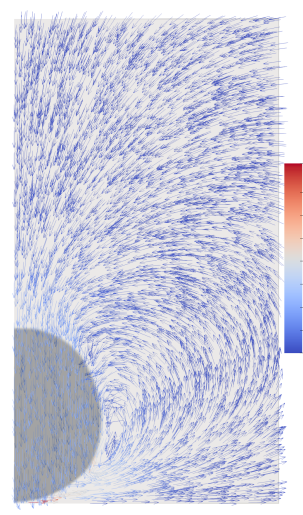

$0.0 \mathrm{~ms}$

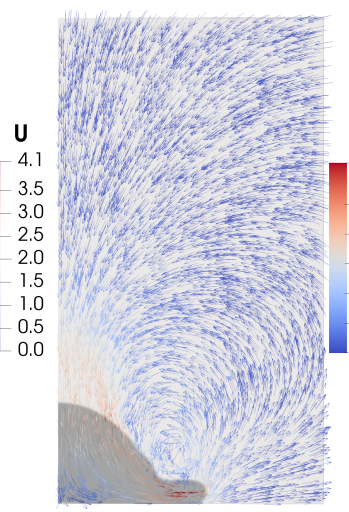

$1.0 \mathrm{~ms}$

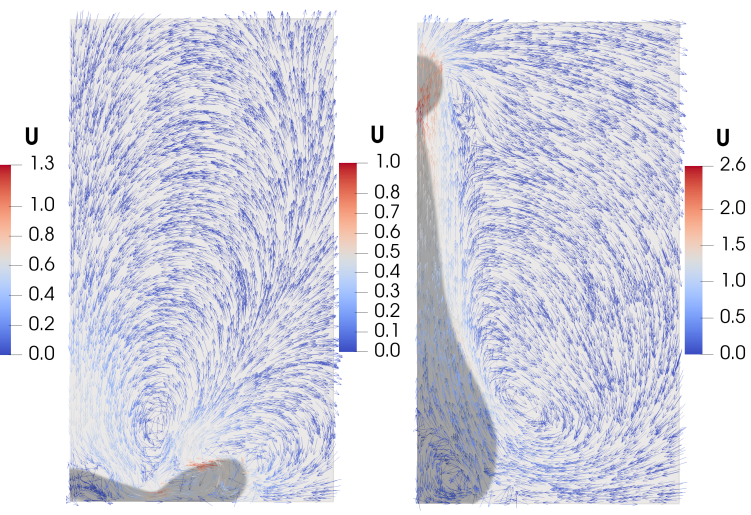

$3.0 \mathrm{~ms}$

(a)

$\mathrm{U}$
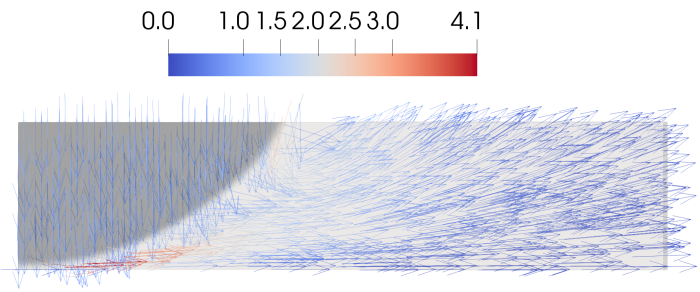

$0.0 \mathrm{~ms}$

U

$\begin{array}{llllll}0.0 & 0.2 & 0.4 & 0.6 & 0.8 & 1.0\end{array}$

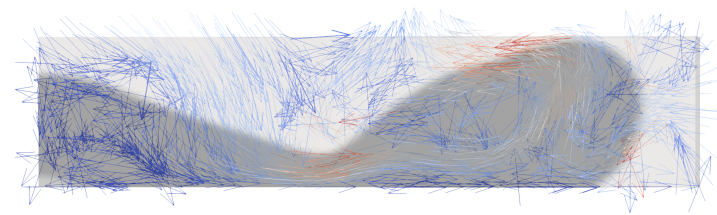

$3.0 \mathrm{~ms}$

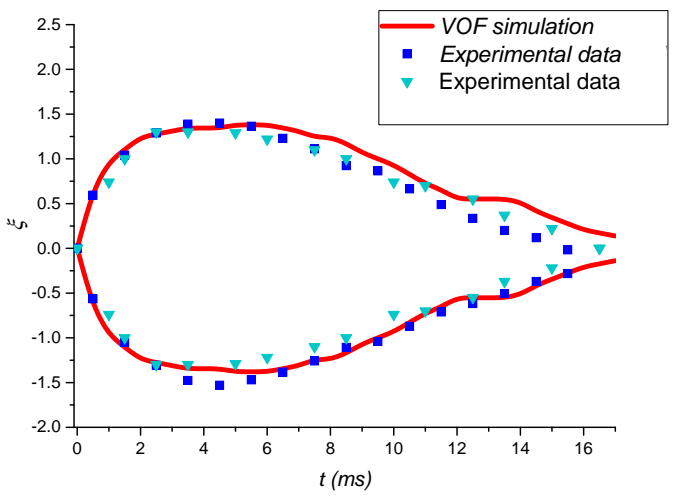

(c)
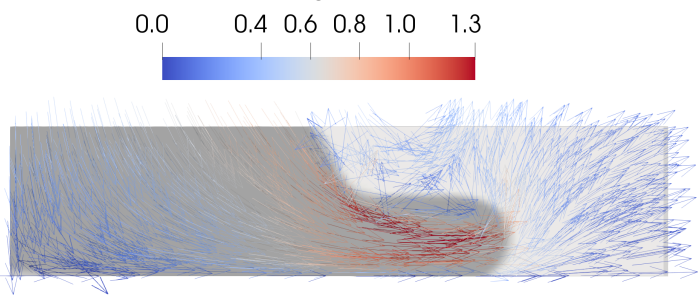

$1.0 \mathrm{~ms}$

U

$\begin{array}{lll}0.0 & 0.20 .30 .40 .50 .60 .7 & 0.9\end{array}$

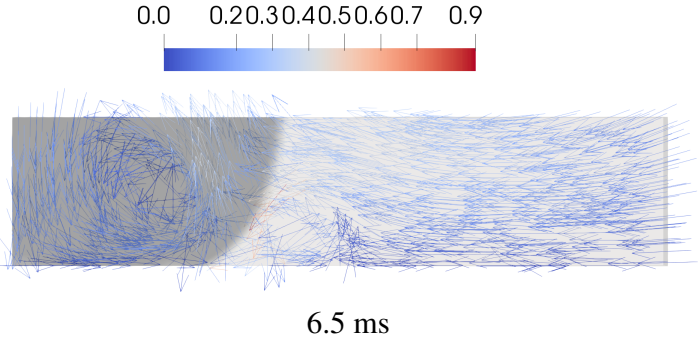

$6.5 \mathrm{~ms}$

(b)

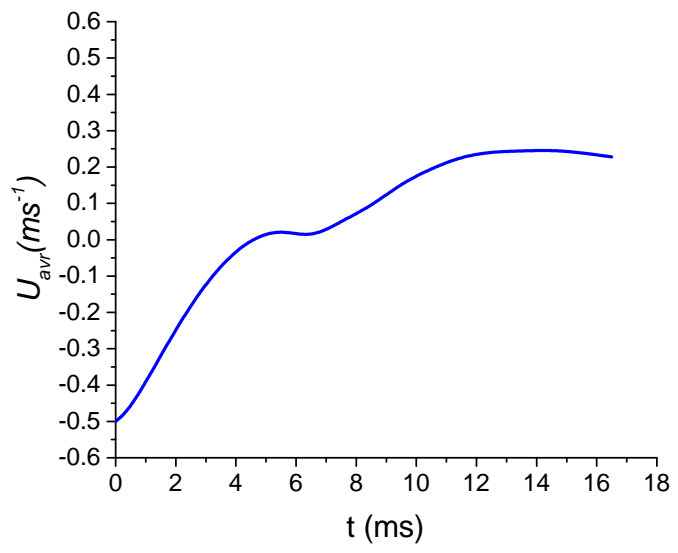

(d)

FIG. 5. (a) Simulated dynamic droplet shapes with $D_{\circ}=2.1 \mathrm{~mm}, U_{\circ}=0.5 \mathrm{~ms}^{-1}$, and $\theta_{0}=\theta_{a}=\theta_{r}=150^{\circ}(W e=7.5$ and $R e=1050)$. The droplet shape demonstrates the expected behaviors of impact, spreading, receding and rebound. (b) Velocity field near the contact line. (c) The contact line of the simulated droplet impact (red line) is compared to independently-reported experimental results with similar impact conditions. Apart from a slight asymmetry for the experimental data, there is fairly good agreement between the data sets; the experimental data were taken from Schutzius et al. [25] (squares) and Li et al. [88] (triangles). (d) Predicted average droplet velocity as a function of time after initial contact. This quantity is difficult to measure experimentally, but it can be evaluated with relative ease numerically. 


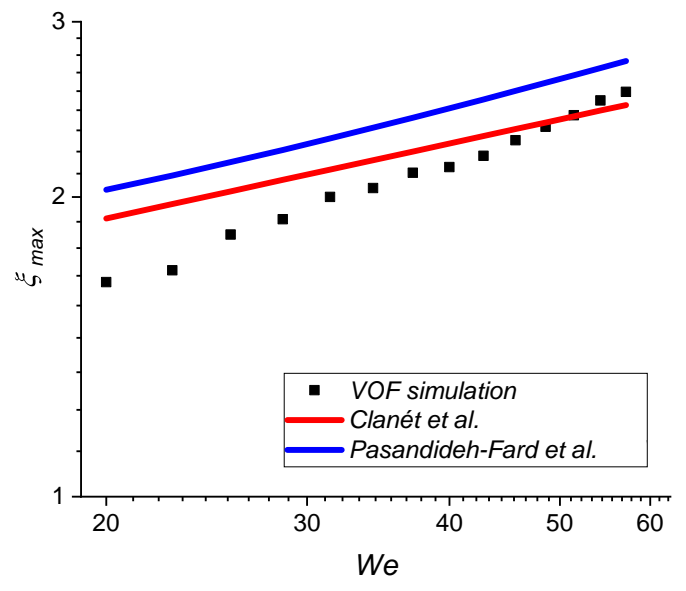

(a)

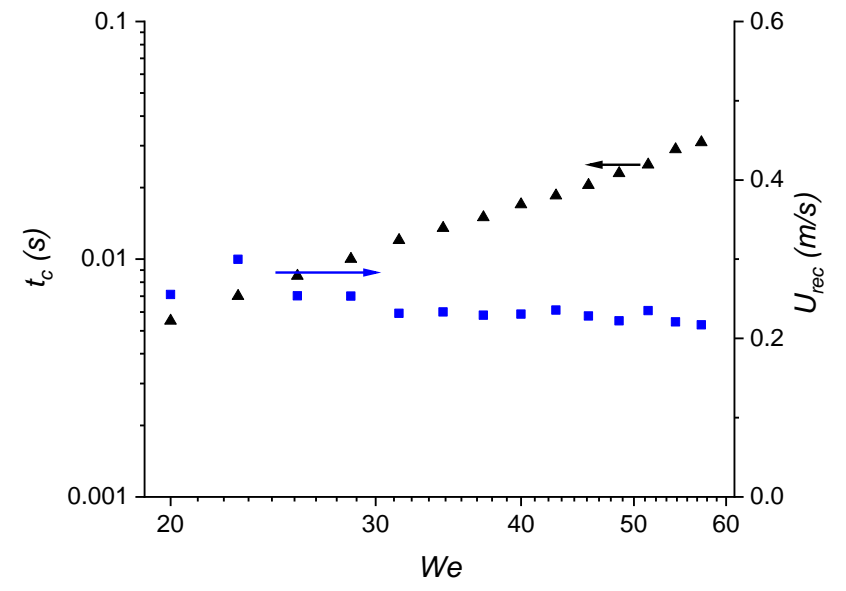

(b)

FIG. 6. (a) Numerically-predicted maximum spreading factors for droplets with same initial velocity $U_{\circ}$ and varying initial diameter $D_{\circ}$ striking orthogonally a superhydrophobic surface. Numerical results on average show a $13 \%$ difference from the predictions of the model of Pasandideh-Fard et al. [14], and an average 6\% difference from the predictions of Clanét et al. [17], the latter derived specifically for superhydrophobic surfaces. (b) Contact time (black triangles) and average receding velocity (blue squares) of droplets impacting with constant velocity, $U_{\circ}$, and varying diameter $D_{\circ}$. In agreement with the model of Richard et al. [85], the dependence of the contact time on $D_{\circ}$ is clear. Moreover, as predicted by Bartolo et al. [87], the average receding velocity is proportional to the initial velocity (thus, in this case the receding velocity remained almost constant). In the Supplementary Material, we report data on maximum spreading factor, contact time and average receding velocity for impacts with the same initial diameter $D_{\circ}$ and varying initial velocity $U_{\circ}$.

\section{B. Impact on spatially non-uniform surfaces}

After validating the numerical model for impact on uniformly-superhydrophobic and hydrophilic surfaces, we studied impact on non-uniform (in terms of wettability) surfaces. These consist of combinations of hydrophilic and superhydrophobic [89] domains of similar properties to those tested above. We consider designs previously reported and demonstrating droplet translation, vectoring, and volume splitting. In addition, we explore a new wettability pattern that facilitates droplet entrapment.

\section{Droplet impact on the boundary line between hydrophilic and superhydrophobic semi-infinite regions}

This non-axisymmetric case considers a droplet impacting on a straight line separating two semi-infinite regions with different wettabilities. The regions feature hydrophilic and superhydrophobic properties, respectively, and the droplet impact occurs with $D_{0}=2.1 \mathrm{~mm}$ and $U_{0}=1.41 \mathrm{~ms}^{-1}$. Figure 7 a shows how each region interacts with the impacting liquid. More importantly, the maximum spreading on the hydrophilic side is equivalent to that for a uniformly-hydrophilic surface. Similarly, the maximum spreading on the superhydrophobic side is equivalent to that predicted for impact on a uniformly-superhydrophobic surface. Intuitively, this outcome makes sense, even though the droplet is impacting on a MRS; in this circumstance, the liquid over each region does not feel the corresponding effects of the other half region, and thus the liquid spreading unravels as if each surface were on its own. After maximal lateral spreading, the superhydrophobic surface also facilitates receding. With the droplet pinned over the hydrophilic domain, the energy from the receding fluid is directed from the superhydrophobic to the hydrophilic side, and eventually brings the entire fluid into the hydrophilic region. This behavior can be seen in greater detail in the contact line graph presented in Figure 7b. The images in Fig. 7a show that even though the droplet remains pinned as a whole, there is enough energy remaining in the receding fluid to induce a partial rebound [90].

In Figure 8, we plot the positions of the contact line at the maximum spreading condition as function of the contact angle difference $(C A D)$, namely the difference between the contact angles of the two domains. For this impact, the results show that the values of the maximum spreading diameter are independent of $C A D$, but a shift of the droplet center towards the hydrophilic substrate is induced by the non-uniform wettability conditions. 


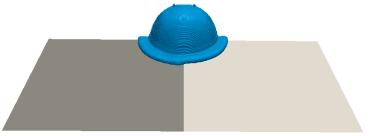

$0.5 \mathrm{~ms}$

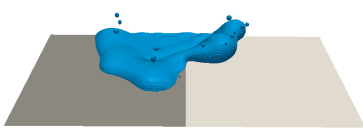

$4.5 \mathrm{~ms}$

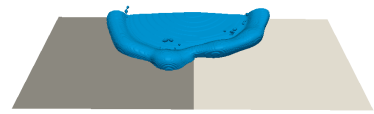

$2.5 \mathrm{~ms}$

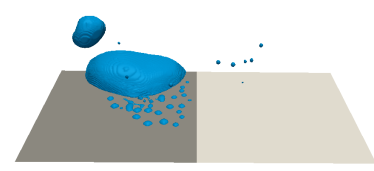

$16 \mathrm{~ms}$

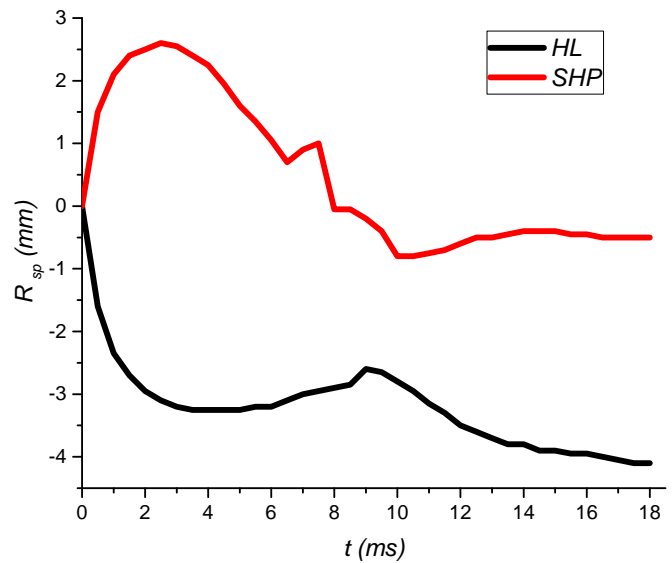

(b)

(b)

(a)

FIG. 7. (a) Droplet impact on a wettability step. The dark-grey region (hydrophilic) has contact angles of $\theta_{0}=89^{\circ}, \theta_{a}=93^{\circ}, \theta_{r}=0^{\circ}$, while the light-grey region (superhydrophobic) has $\theta_{0}=159^{\circ}, \theta_{a}=166^{\circ}, \theta_{r}=155^{\circ}$. As observed from the impact snapshots (a) and the contact-line graph (b), each portion of the impacting droplet spreads on its respective region, independent of what occurs over the opposite side. Once full spreading has been reached, the superhydrophobic (SHP) region allows the fluid to recede, while the fluid over the hydrophilic (HL) region remains pinned. The droplet portion on the superhydrophobic region gradually recedes until it is entirely drawn over the hydrophilic region, sometimes causing secondary-droplet departure.

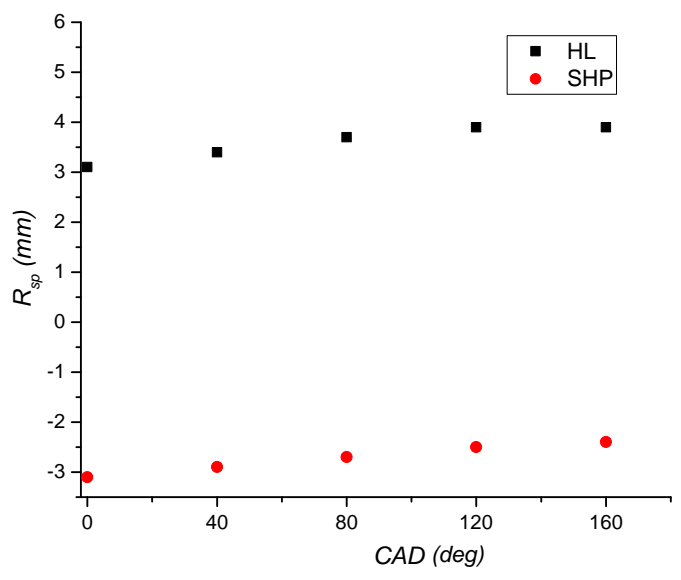

FIG. 8. Maximum spreading of a droplet striking a wettability step with increasing contact angle difference $(C A D)$ on either side of the step. Specifically, null $C A D$ corresponds to the uniform-wettability case, i.e. when both sides have contact angles of $\theta_{0}=\theta_{a}=\theta_{r}=90^{\circ}$, while the non-zero $C A D$ corresponds to hydrophilic (HL) and hydrophobic (SHP) sides with $\theta_{0}=\theta_{a}=\theta_{r}=90^{\circ}-C A D / 2$ and $\theta_{0}=\theta_{a}=\theta_{r}=$ $90^{\circ}+C A D / 2$, respectively.

\section{Droplet impact on a hydrophilic substrate with a superhydrophobic strip}

Splitting droplets into pre-specified volumes without external energy expenditure is an important capability that holds application possibilities in the realm of digital microfluidics. We use our numerical tools and wettability-patterned surfaces to induce volume splitting in impacting droplets. To this end, we simulate droplet impact on a surface with a superhydrophobic strip surrounded by semi-infinite hydrophilic regions. As previously reported in Schutzius et al. [25], two observed outcomes are possible: for wider strips, a partial droplet rebound occurs, while for narrower strips, the droplet splits into two parts, each lying entirely on the respective hydrophilic surface with no partial volume rebound.

Figures 9a and 9b show the simulated impact on the center of $3 \mathrm{~mm}$ and $0.2 \mathrm{~mm}$-wide superhydrophobic strips, respectively. 
It can be seen that a partial rebound of the droplet occurs on the wider $(3 \mathrm{~mm})$ strip, Figure $9 \mathrm{a}$, but not on the $0.2 \mathrm{~mm}$ strip (Figure 9b). This observation is consistent with the experiments of Schutzius et al. [25]. The maximum spreading factor and the droplet shape during the impact are also evaluated numerically, and are in agreement with those observed experimentally. It is interesting to notice that the spreading factor evaluated for the direction parallel to the strip follows a trend typical of impacts on superhydrophobic surfaces. On the other hand, the spreading factor along the direction perpendicular to the strip, after maximum spreading, starts decreasing until it reaches a minimum value exactly at detachment of the rebounded droplet, and then it starts rising again during the relaxation phase [91]. Figure 9c shows that the maximum spreading radii evaluated at the superhydrophobic and the hydrophilic surfaces do not depend on the strip widths.

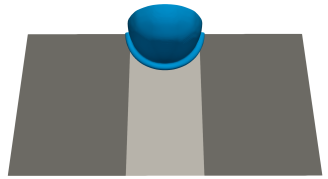

$0.5 \mathrm{~ms}$

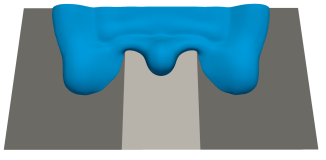

$8 \mathrm{~ms}$

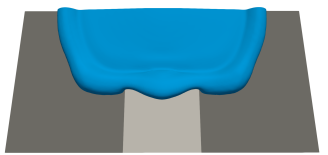

$4.5 \mathrm{~ms}$

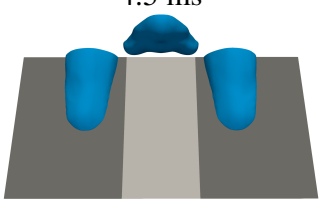

Final

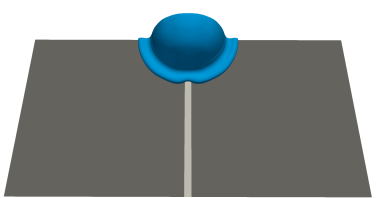

$0 \mathrm{~ms}$

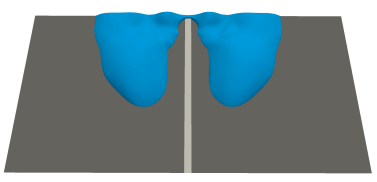

$7 \mathrm{~ms}$

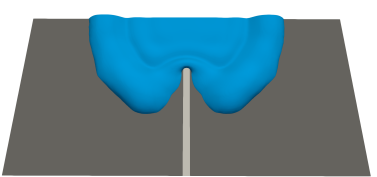

$4.5 \mathrm{~ms}$

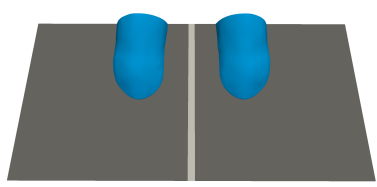

Final (a)

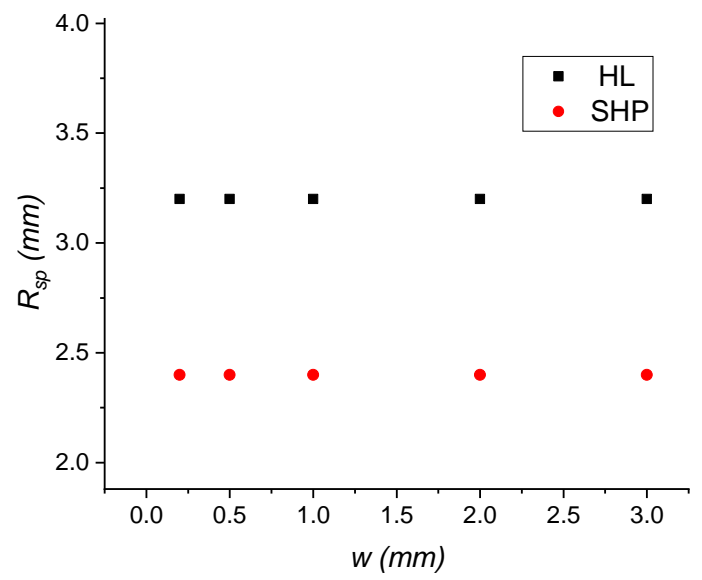

(b)

(c)

FIG. 9. Droplet impact $(W e=60)$ on wettability-patterned surfaces (WPS) consisting of hydrophilic $\left(\theta_{0}=89^{\circ}, \theta_{a}=93^{\circ}\right.$ and $\left.\theta_{r}=0^{\circ}\right)$ areas (in dark grey) surrounding superhydrophobic $\left(\theta_{0}=159^{\circ}, \theta_{a}=166^{\circ}, \theta_{r}=155^{\circ}\right)$ strips (in light grey) of width: (a) $3 \mathrm{~mm}$, and (b) $0.2 \mathrm{~mm}$. The width of the superhydrophobic strip controls the ultimate shape of the impacting liquid, and residual liquid volume rebounded from the surface. The same dynamic phenomena were previously observed experimentally by Schutzius et al. [25]. (c) Maximum spreading factor for the superhydrophobic and hydrophilic surfaces for varying strip widths $w$.

\section{Droplet impact on a superhydrophobic substrate with a hydrophilic arc}

Proceeding to more complex configurations, we simulated droplet impact on a superhydrophobic surface with a thin hydrophilic circular arc, having external radius $r$, approximately equal to the maximum spreading droplet radius. Analogous to experiments performed by Schutzius et al. [25], the simulated impact occurred at $W e=100$. As seen in Fig. 10a, the model predictions revealed non-orthogonal rebound, as also observed experimentally. The pinning of the liquid on the arc causes an asymmetric receding behavior, which results in a net transfer of momentum in the horizontal direction, yielding rebound in non purely-vertical direction. As seen in Figure 10b, the leftmost contact line remains pinned on the philic arc as the rightmost contact line continues to recede inwards. Once the rightmost contact line traverses the arc center $\left(R_{\mathrm{sp}}=0\right)$, the droplet begins its rebound. Due to the pinning of the leftmost contact line, the receding contact line is forced to recede past the impact point 
$\left(R_{\mathrm{sp}}=0\right)$. This results in a prolonged contact time, and increased energy loses (refer to Supplementary Information for a link to the full simulation video $V 3: A r c$ ).

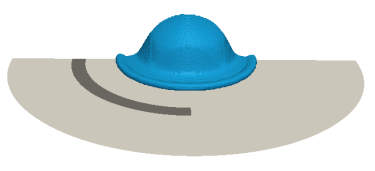

$0.5 \mathrm{~ms}$

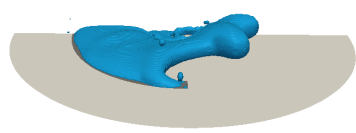

$5.5 \mathrm{~ms}$

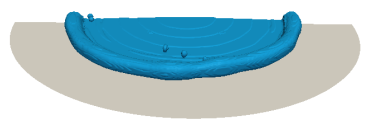

$2.5 \mathrm{~ms}$

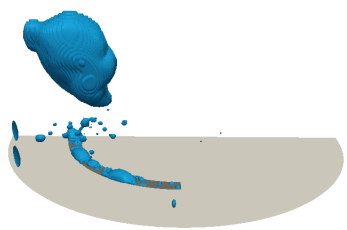

$14 \mathrm{~ms}$

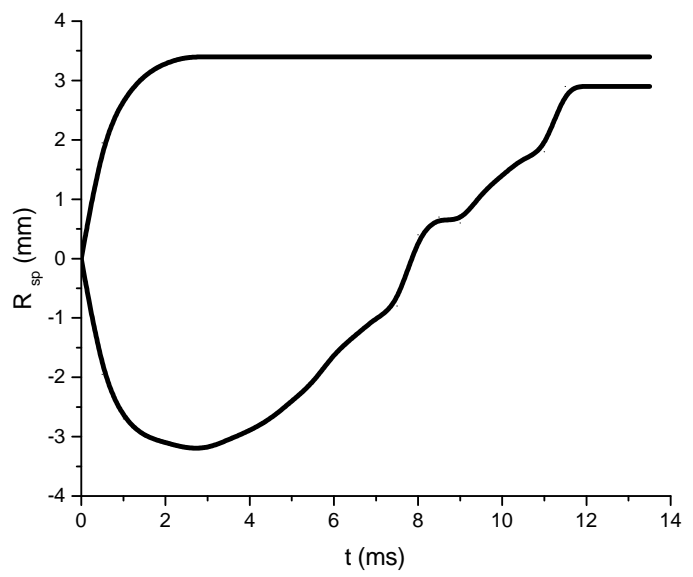

(b)

FIG. 10. (a) Simulation of droplet impacting orthogonally with $W e=100\left(D_{0}=2.2 \mathrm{~mm}, U_{0}=1.83 \mathrm{~ms}^{-1}\right)$ on a superhydrophobic surface $\left(\theta_{0}=159^{\circ}, \theta_{a}=166^{\circ}, \theta_{r}=155^{\circ}\right)$ with a hydrophilic arc $\left(\theta_{0}=89^{\circ}, \theta_{a}=93^{\circ}\right.$ and $\left.\theta_{r}=0^{\circ}\right)$ of external radius $r=3.29$ mm and width $w=0.4 \mathrm{~mm}$. As seen in the shapes in (a) and the temporal variation of the two contact-line radii on the symmetry axis in (b), the impacting droplet spreads, becomes pinned onto the hydrophilic arc, and eventually recedes, and rebounds in a non-orthogonal fashion (event known as vectoring).

\section{Droplet impact on center of disk surrounded by a different-wettability area}

We also simulated orthogonal impact at the center of a circular disk of specific wettability and size, and surrounded by a semi-infinite region of different wettability. The corresponding droplet behaviors during impact under two circumstances were studied: 1) Hydrophilic disk in superhydrophobic exterior, and 2) superhydrophobic disk in hydrophilic surroundings. The contact angles are $\theta_{0}=\theta_{a}=\theta_{r}=150^{\circ}$ in the superhydrophobic regions, and $\theta_{0}=\theta_{a}=\theta_{r}=60^{\circ}$ in the hydrophilic domains. The impact conditions were kept constant, and the diameter $w$ of the disk was varied. The numerically-calculated maximum spreading factors for each case are plotted in Figure 11.

For the case of a superhydrophobic disk in hydrophilic surroundings, Fig. 11a shows that for smaller disk diameters, the maximum spread is comparable to that predicted for a droplet striking a uniformly-hydrophilic area. This can been seen in the graph, where the blue line is the predicted spreading factor for a droplet impacting on a uniform hydrophilic region of the same wettability and impact conditions. This implies that the spreading behavior is independent of the original superhydrophobic disk, and only dependent on the outside hydrophilic area. A reason for this outcome is that the superhydrophobic area is not large enough to have a significant impact on the spreading behavior. In contrast, as the diameter of the disk increases, the model results deviate from the predictions of Pasandideh-Fard et al. [14]. Eventually, for large enough disks, the observed maximum spreading diameter is no longer predicted by Pasandideh-Fard et al.'s model, but rather the model of Clanét et al. [17] for a uniformlysuperhydrophobic surface (green line). In the latter case, the disk is large enough to accommodate the entire spreading range, which now occurs entirely within the superhydrophobic area.

Next, we studied central impact on a hydrophilic disk in superhydrophobic surroundings. As seen from Figure 11b, for smaller disks, the observed spreading factor can be predicted using the Clanét model for a superhydrophobic surface. This observation can be explained using the same logic presented above; the area of the hydrophilic disk is small enough so that it does not exert a significant influence on the droplet spreading behavior when compared to the surrounding superhydrophobic region. As the disk diameter increases, an interesting situation occurs wherein the observed maximum spreading is equivalent to the disk diameter (linear portion of Figure 11b). In other words, for such conditions, a spreading droplet becomes entrapped within the hydrophilic disk and is unable to spread farther onto the superhydropobic surroundings. This physical entrapment is interesting and intuitive. The outside superhydrophobic region acts as an energy barrier, preventing the spreading droplet from transitioning into it. This phenomenon should be observed not only for hydrophilic/superhydrophobic contrasts, but also for other situations where the surrounding region is less wettable than the inner disk. As the diameter of the disk increases, the observed spreading 
factors begin to fall in with the predictions of Pasandideh-Fard et al.'s model for impact on a uniformly-hydrophilic surface. At this point, the diameter of the disk has become so large, that the entire spreading is confined within the hydrophilic region.

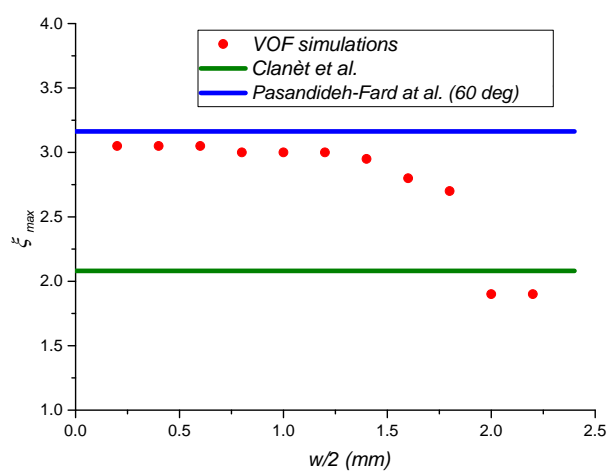

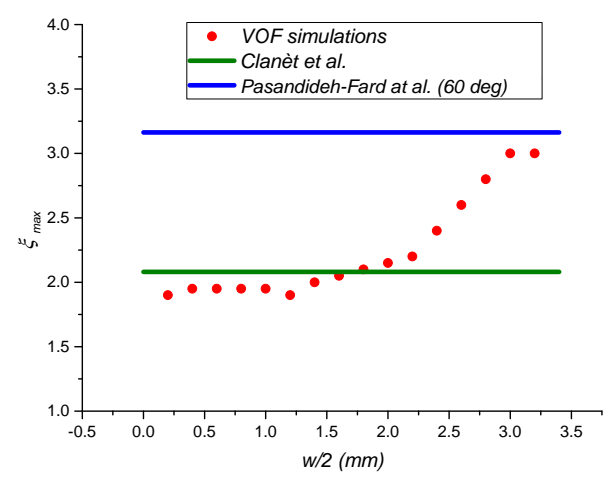

(a) (b)

FIG. 11. Maximum spreading factor evaluated with the present VOF numerical model for central and orthogonal droplet impact with $W e \approx 60$ on: (a) a superhydrophobic disk in hydrophilic surroundings, and (b) a hydrophilic disk in superhydrophobic surroundings. The superhydrophobic domains have contact angles $\theta_{0}=\theta_{a}=\theta_{r}=150^{\circ}$, while the hydrophilic ones have contact angles $\theta_{0}=\theta_{a}=\theta_{r}=60^{\circ}$. The impact diameter and the impact velocity are $D_{0}=2.1 \mathrm{~mm}$ and $U_{0}=1 \mathrm{~ms}^{-1}$, respectively, and the disk diameter is $w$. We report also the maximum spreading diameters obtained with the models proposed by Pasandideh-Fard et al. [14] (blue line) and by Clanét et al. [17] (green line).

\section{CONCLUSIONS}

The realm of wettability-patterned surfaces is vast, with nearly limitless design combinations of different shapes, surface energies and textures. Relying only on experimental investigations for testing such surfaces would be infeasible and expensive. In this work, we have presented a numerical method capable of simulating droplet impact on such spatially-inhomogeneous planar surfaces. We began by benchmarking our numerical simulations against the commonly-accepted models of Pasandideh-Fard et al. [14] and Clanét et al. [17], and found strong agreement in select problems where the solid surface wettability is spatially uniform. Next, we examined droplet impact on wettability-patterned surfaces capable of producing droplet translation, splitting, and vectoring. In the case of translation, we studied a droplet impacting orthogonally a wettability step and moving laterally until equilibrating into the region of higher surface energy. For splitting, we showed how the width of a hydrophobic strip surrounded by hydrophilic areas dictates the size of the liquid-volume split, and the residual volume that rebounds. Lastly, we simulated the non-orthogonal rebound (vectoring) of droplets impacting orthogonally on a superhydrophobic domain with a hydrophilic circular arc, which serves as an anchoring site. Our simulation results agreed with the data reported by Schutzius et al. [25], where the same patterns were experimentally tested and analyzed. Furthermore, we simulated cases where spreading droplets that impacted orthogonally a disk of specific wettability became entrapped by the surrounding region that had lower wettability. Although the present model has been vetted with a limited number of wettability designs, the techniques presented here are amenable to any foreseeable wettability-patterned surface. Moreover, following the recent experimental work by Jambon-Puillet et al. [92], the presented methodology can be extended to study numerically the deformation of elastic capsules filled with different liquids impacting rigid walls.

\section{ACKNOWLEDGMENTS}

Computational resources were mainly provided by HPC@Polito, a project of academic computing within the Department of Control and Computer Engineering at the Politecnico di Torino (http://www.hpc.polito.it). MI and DC gratefully acknowledge the support of Raúl Tempone, the KAUST SRI-UQ center, and the KAUST HPC and Extreme Computing center. M.E. was supported by a National Science Foundation Graduate Research Fellowship, under Grant No. 0907994.

[1] M. Rein, "Phenomena of liquid drop impact on solid and liquid surfaces," Fluid Dyn. Res. 12, 61 (1993). 
[2] M. Marengo, C. Antonini, I. V. Roisman, and C. Tropea, "Drop collisions with simple and complex surfaces," Curr. Opin. Colloid Interface Sci. 16, 292 - 302 (2011).

[3] C. Josserand and S. T. Thoroddsen, "Drop impact on a solid surface," Annu. Rev. Fluid Mech. 48, 365-391 (2016).

[4] B. Derby, "Inkjet printing of functional and structural materials: Fluid property requirements, feature stability, and resolution," Annu. Rev. Mater. Res. 40, 395-414 (2010).

[5] S. Jung, S. D. Hoath, and I. M. Hutchings, "The role of viscoelasticity in drop impact and spreading for inkjet printing of polymer solution on a wettable surface," Microfluid. Nanofluid. 14, 163-169 (2013).

[6] R. Andrade, O. Skurtys, and F. Osorio, "Drop impact behavior on food using spray coating: Fundamentals and applications," Food Res. Int. 54, $397-405$ (2013).

[7] S.-S.Hsieh and S.-Y. Luo, "Droplet impact dynamics and transient heat transfer of a micro spray system for power electronics devices," Int. J. Heat Mass Trans. 92, 190 - 205 (2016).

[8] S.-P. Fu, R. P. Sahu, E. Diaz, J. R. Robles, C. Chen, X. Rui, R. F. Klie, A. L. Yarin, and J. T. Abiade, "Dynamic study of liquid drop impact on supercooled cerium dioxide: Anti-icing behavior," Langmuir 32, 6148-6162 (2016).

[9] R. Blossey, "Self-cleaning surfaces - virtual realities," Nat. Mater. 2, 301-306 (2003).

[10] J. Ma, X. Y. Zhang, D. P. Wang, D. Q. Zhao, D. W. Ding, K. Liu, and W. H. Wang, "Superhydrophobic metallic glass surface with superior mechanical stability and corrosion resistance," Appl. Phys. Lett. 104, 173701 (2014).

[11] D. C. Vadillo, A. Soucemarianadin, C. Delattre, and D. C. D. Roux, "Dynamic contact angle effects onto the maximum drop impact spreading on solid surfaces," Phys. Fluids 21, 122002 (2009).

[12] H. Y. Kim and J. H. Chun, "The recoiling of liquid droplets upon collision with solid surfaces," Phys. Fluids 13, 643-659 (2001).

[13] D. Bartolo, F. Bouamrirene, E. Verneuil, A. Buguin, P. Silberzan, and S. Moulinet, "Bouncing or sticky droplets: Impalement transitions on superhydrophobic micropatterned surfaces," Europhys. Lett. 74, 299 (2006).

[14] M. Pasandideh-Fard, Y. M. Qiao, S. Chandra, and J. Mostaghimi, "Capillary effects during droplet impact on a solid surface," Phys. Fluids 8, 650-659 (1996).

[15] B. L. Scheller and D. W. Bousfield, "Newtonian drop impact with a solid surface," AIChE J. 41, 1357-1367 (1995).

[16] I. V. Roisman, "Inertia dominated drop collisions. II. an analytical solution of the navier-stokes equations for a spreading viscous film," Phys. Fluids 21, 052104 (2009).

[17] C. Clanét, C. Béguin, R. Denis, and D. Quéré, “Maximal deformation of an impacting drop,” J. Fluid Mech. 517, 199-208 (2004).

[18] A. L. Yarin, "Drop impact dynamics: Splashing, spreading, receding, bouncing," Annu. Rev. Fluid Mech. 38, 159-192 (2006).

[19] V. Vaikuntanathan and D. Sivakumar, "Effect of wetting difference across junction on dynamics of drops impacting on the junction of dual-textured surfaces," AIChE J. 62, 4109-4118 (2016).

[20] M. Lee, Y. S. Chang, and H.-Y. Kim, "Drop impact on microwetting patterned surfaces,” Phys. Fluids 22, 072101 (2010).

[21] S. Kim, M.-W. Moon, and H.-Y. Kim, “Drop impact on super-wettability-contrast annular patterns,” J. Fluid Mech. 730, 328-342 (2013).

[22] J. J. Huang, H. Huang, and X. Wang, "Numerical study of drop motion on a surface with stepwise wettability gradient and contact angle hysteresis," Phys. Fluids 26, 062101 (2014).

[23] T. Michel, U. Mock, I. Roisman, J. Ruehe, and C. Tropea, "The hydrodynamics of drop impact onto chemically structured surfaces," J. Phys. Condens. Matter 17, S607 (2005).

[24] U. Mock, T. Michel, C. Tropea, I. Roisman, and J. Rühe, "Drop impact on chemically structured arrays,” J. Phys. Condens. Matter 17, S595 (2005).

[25] T. M. Schutzius, G. Graeber, M. Elsharkawy, J. Oreluk, and C. M. Megaridis, "Morphing and vectoring impacting droplets by means of wettability-engineered surfaces," Sci. Rep. 4, 7029 (2014).

[26] C. Guo, D. Zhao, Y. Sun, M. Wang, and Y. Liu, "Droplet impact on anisotropic superhydrophobic surfaces," Langmuir 34, 3533-3540 (2018).

[27] B. Farshchian, J. Pierce, M. S. Beheshti, S. Park, and N. Kim, "Droplet impinging behavior on surfaces with wettability contrasts," Microelectron. Eng. 195, 50 - 56 (2018).

[28] B. A. Malouin, N. A. Koratkar, A. H. Hirsa, and Z. Wang, "Directed rebounding of droplets by microscale surface roughness gradients," Appl. Phys. Lett. 96, 234103 (2010).

[29] J. Liu, H. Guo, B. Zhang, S. Qiao, M. Shao, X. Zhang, X.-Q. Feng, Q. Li, Y. Song, L. Jiang, and J. Wang, "Guided self-propelled leaping of droplets on a micro-anisotropic superhydrophobic surface," Angew. Chem. 55, 4265-4269 (2016).

[30] M. Reyssat, F. Pardo, and D. Quéré, “Drops onto gradients of texture,” Europhys. Lett. 87, 36003 (2009).

[31] J. E. Sprittles and Y. D. Shikhmurzaev, "The dynamics of liquid drops and their interaction with solids of varying wettabilities," Phys. Fluids 24, 082001 (2012).

[32] J. J. Huang, C. Shu, and Y. T. Chew, "Numerical investigation of transporting droplets by spatiotemporally controlling substrate wettability," J. Colloid Interface Sci. 328, 124-133 (2008).

[33] M. Chakraborty, A. Chowdhury, R. Bhusan, and S. DasGupta, "Molecular dynamic approach of enhanced self-propelled nano-droplet motion on wettability gradient surfaces," arXiv preprint arXiv:1504.02567 (2015).

[34] J. D. Halverson, C. Maldarelli, A. Couzis, and J. Koplik, "A molecular dynamics study of the motion of a nanodroplet of pure liquid on a wetting gradient," J. Chem. Phys. 129, 164708 (2008).

[35] J. Xu, Y. Chen, and J. Xie, "Non-dimensional numerical study of droplet impacting on heterogeneous hydrophilicity/hydrophobicity surface," Int. J. Heat Mass Trans. 116, 951 - 968 (2018).

[36] X. Wang, D.-L. Sun, X.-D. Wang, and W.-M. Yan, "Dynamics of droplets impacting hydrophilic surfaces decorated with a hydrophobic strip,” Int. J. Heat Mass Trans. 135, 235 - 246 (2019).

[37] H. Li, W. Fang, Y. Li, Q. Yang, M. Li, Q. Li, X.-Q. Feng, and Y. Song, "Spontaneous droplets gyrating via asymmetric self-splitting on heterogeneous surfaces," Nat. Commun. 10, 950 (2019). 
[38] H. G. Weller, G. Tabor, H. Jasak, and C. Fureby, "A tensorial approach to computational continuum mechanics using object-oriented techniques," Comput. Phys. 12, 620-631 (1998).

[39] C. J. Greenshields, “Openfoam programmer's guide," OpenFOAM documentation (2005).

[40] S Márquez Damián, An extended mixture model for the simultaneous treatment of short and long scale interfaces, Ph.D. thesis, Universidad Nacional del Litoral (2013).

[41] H.B. Parizi, L. Rosenzweig, J. Mostaghimi, S. Chandra, T. Coyle, H. Salimi, L. Pershin, A. McDonald, and C. Moreau, "Numerical simulation of droplet impact on patterned surfaces," J. Therm. Spray Technol. 16, 713-721 (2007).

[42] R. Zhang, S. Farokhirad, T. Lee, and J. Koplik, "Multiscale liquid drop impact on wettable and textured surfaces," Phys. Fluids 26, 082003 (2014).

[43] A. Dupuis, A. J. Briant, C. M. Pooley, and J. M. Yeomans, "Droplet spreading on heterogeneous surfaces using a three-dimensional lattice boltzmann model," in Computational Science - ICCS 2003: International Conference, Melbourne, Australia and St. Petersburg, Russia, June 2-4, 2003 Proceedings, Part I, edited by Peter M. A. Sloot, David Abramson, Alexander V. Bogdanov, Jack J. Dongarra, Albert Y. Zomaya, and Yuriy E. Gorbachev (Springer Berlin Heidelberg, Berlin, Heidelberg, 2003) pp. 1024-1033.

[44] F. H. Harlow and J. P. Shannon, "The splash of a liquid drop," J. Appl. Phys. 38, 3855-3866 (1967).

[45] B. J. Daly, "Numerical study of two fluid rayleigh-taylor instability," Phys. Fluids 10, 297-307 (1967).

[46] I. Malgarinos, N. Nikolopoulos, M. Marengo, C. Antonini, and M. Gavaises, "Vof simulations of the contact angle dynamics during the drop spreading: Standard models and a new wetting force model," Adv. Colloid Interface Sci. 212, 1 - 20 (2014).

[47] H. Liu, E. J. Lavernia, and R. H. Rangel, "Numerical simulation of substrate impact and freezing of droplets in plasma spray processes," J. Phys. D 26, 1900 (1993).

[48] J. P. Boris and D. L. Book, "Flux-corrected transport. i. shasta, a fluid transport algorithm that works," J. Comp. Phys. 11, 38 - 69 (1973).

[49] M. Bussmann, S. Chandra, and J. Mostaghimi, "Modeling the splash of a droplet impacting a solid surface," Phys. Fluids 12, 3121-3132 (2000).

[50] P. R. Gunjal, V. V. Ranade, and R. V. Chaudhari, "Dynamics of drop impact on solid surface: Experiments and VOF simulations," AIChE J. 51, 59-78 (2005).

[51] S. Osher and J. A. Sethian, "Fronts propagating with curvature dependent speed: Algorithms based on hamilton-jacobi formulations," J. Comp. Phys. 79, 12-49 (1988).

[52] N. D. Patil, V. H. Gada, and A. Sharma, "On dual-grid level-set method for contact line modeling during impact of a droplet on hydrophobic and superhydrophobic surfaces," Int. J. Multiph. Flow 81, 54-66 (2016).

[53] J. Fukai, Z. Zhao, D. Poulikakos, C. M. Megaridis, and O. Miyatake, "Modeling of the deformation of a liquid droplet impinging upon a flat surface," Phys. Fluids A 5, 2588-2599 (1993).

[54] S. Mukherjee and J. Abraham, "Investigations of drop impact on dry walls with a lattice-boltzmann model," J. Colloid Interface Sci. 312, 341-354 (2007).

[55] A. Fakhari and M. H. Rahimian, "Simulation of falling droplet by the lattice boltzmann method," Comm. Nonlinear Sci. 14, 3046 - 3055 (2009).

[56] C. W. Hirt and B. D. Nicholson, "Volume of fluid (vof) method for the dynamics of free boundaries,” J. Comp. Phys. 39, 201-225 (1981).

[57] J. U. Brackbill, D. B. Kothe, and C. Zemach, “A continuum method for modeling surface tension,” J. Comp. Phys. 100, 335 - 354 (1992).

[58] J. Klostermann, K. Schaake, and R. Schwarze, "Numerical simulation of a single rising bubble by vof with surface compression," Int. J. Numer Methods Fluids 71, 960-982 (2013).

[59] S. Patankar, Numerical Heat Transfer and Fluid Flow (CRC Press, 1980).

[60] H. K. Versteeg and W. Malalasekera, An introduction to computational fluid dynamics: the finite volume method (Pearson Education, 2007).

[61] H. Jasak, Error Analysis and Estimation for Finite Volume Method with Applications to Fluid Flow, Ph.D. thesis, Imperial College London (1996).

[62] C. Rhie and W. Chow, "Numerical study of the turbulent flow past and airfoil with trailing edge separation," AIAA J. 21, 1525-1532 (1983).

[63] F. P. Kärrholm, "Rhie-chow interpolation in openfoam,” (2006).

[64] B. van Leer, "Towards the ultimate conservative difference scheme. iv. a new approach to numerical convection," J. Comp. Phys. 23, 276 -299 (1977).

[65] B. van Leer, "Towards the ultimate conservative difference scheme. v. a second-order sequel to godunov's method," J. Comp. Phys. 32, $101-136$ (1979).

[66] R. I. Issa, "Solution of the implicitly discretised fluid flow equations by operator-splitting," J. Comp. Phys. 62, 40-65 (1986).

[67] J. H. Ferziger and M. Peric, "Computational methods for fluid dynamics," (1997).

[68] T. Young, “An essay on the cohesion of fluids," Phil. Trans. R. Soc. Lond 95, 65-87 (1805).

[69] I. S. Bayer and C. M. Megaridis, "Contact angle dynamics in droplets impacting on flat surfaces with different wetting characteristics," J. Fluid Mech. 558, 415-449 (2006).

[70] K. Yokoi, D. Vadillo, J. Hinch, and I. Hutchings, "Numerical studies of the influence of the dynamic contact angle on a droplet impacting on a dry surface," Phys. Fluids 21, 072102 (2009).

[71] S. F. Kistler, "Hydrodynamics of wetting," in Wettability (Marcel Dekker, 1993) pp. 311-429.

[72] R. L. Hoffman, "A study of the advancing interface. i. interface shape in liquid-gas systems," J. Colloid Interface Sci. 50, 228 - 241 (1975).

[73] B. P. LeClair, A. E. Hamielec, H. R. Pruppacher, and W. D. Hall, "A theoretical and experimental study of the internal circulation in water drops falling at terminal velocity in air," J. Atmos. Sci. 29, 728-740 (1972).

[74] R. Rioboo, M. Marengo, and C. Tropea, "Time evolution of liquid drop impact onto solid , dry surfaces," Exp. Fluids 33, 112-124 (2002). 
[75] S. Popinet, "Lecture notes introduction to numerical methods for interfacial flows," (2011).

[76] S. S. Deshpande, L. Anumolu, and M. F. Trujillo, "Evaluating the performance of the two-phase flow solver interfoam," Comput. Sci. Discov. 5, 014016 (2012).

[77] C. Galusinski and P. Vigneaux, "On stability condition for bifluid flows with surface tension: Application to microfluidics," J. Comp. Phys. 227, 6140-6164 (2008).

[78] See Supplemental Material at [URL will be inserted by publisher] for an analysis of spurious currents at constant Eo and at constant We and $\mathrm{Ca}$.

[79] D. J. E. Harvie, M. R. Davidson, and M. Rudman, "An analysis of parasitic current generation in volume of fluid simulations," Appl. Math. Model. 30, 1056-1066 (2006).

[80] C. M. Megaridis, J. T. Hodges, J. Xin, J. M. Day, and C. Presser, “Internal droplet circulation induced by surface-driven rotation,” Int. J. Heat Fluid Flow 15, 364-377 (1994).

[81] See Supplemental Material at [URL will be inserted by publisher] for a discussion of OpenFoam solving parameters.

[82] O. Ubbink, Numerical prediction of two fluid systems with sharp interfaces, Tech. Rep. (University of London, 1997).

[83] C. Ukiwe and D. Y. Kwok, "On the maximum spreading diameter of impacting droplets on well-prepared solid surfaces," Langmuir 21, 666-673 (2005).

[84] S. Wildeman, C. W. Visser, C. Sun, and D. Lohse, "On the spreading of impacting drops," J. Fluid Mech. 805, 636-655 (2016).

[85] D. Richard, C. Clanét, and D. Quéré, "Surface phenomena: Contact time of a bouncing drop," Nature 417, 811-811 (2002).

[86] For cases with constant drop diameter but varied impact velocity, see the Supplementary Material at [URL will be inserted by publisher].

[87] D. Bartolo, C. Josserand, and D. Bonn, "Retraction dynamics of aqueous drops upon impact on non-wetting surfaces," J. Fluid Mech. 545, 329-338 (2005).

[88] X. Li, X. Ma, and Z. Lan, "Dynamic behavior of the water droplet impact on a textured hydrophobic/superhydrophobic surface: The effect of the remaining liquid film arising on the pillars' tops on the contact time," Langmuir 26, 4831-4838 (2010).

[89] Unless otherwise stated, all surfaces referred to as hydrophilic/superhydrophobic, possess $\theta_{0}=89^{\circ}, \theta_{a}=93^{\circ}, \theta_{r} \approx 0^{\circ}$, and $\theta_{0}=159^{\circ}$, $\theta_{a}=166^{\circ}, \theta_{r}=155^{\circ}$, respectively.

[90] Refer to Supplemental Material at [URL will be inserted by publisher] for a link to the full simulation video V1:Contrast Line.

[91] Refer to Supplemental Material at [URL will be inserted by publisher] for a link to the full simulation video of impact on the 3 mm strip V2:Strip.

[92] E. Jambon-Puillet, T. J. Jones, and P. T. Brun, “Deformation and bursting of elastic capsules impacting a rigid wall,” Nat. Phys. (2020), 10.1016/j.ijheatmasstransfer.2017.09.068. 